\title{
In situ growth of fluorescent silicon nanocrystals in monolithic microcapsule as a photostable, versatile platform
}

\author{
Guixian Zhu $^{\mathrm{a}, \mathrm{b}}$, Yu Huang ${ }^{\mathrm{c}}$, Gauri Bhave ${ }^{\mathrm{b}}$, Yuzhen Wang ${ }^{\mathrm{b}}$, Zhongbo Hu${ }^{\mathrm{a}}$, and Xuewu Liu ${ }^{\mathrm{b}}$ \\ Xuewu Liu: xliu@houstonmethodist.org

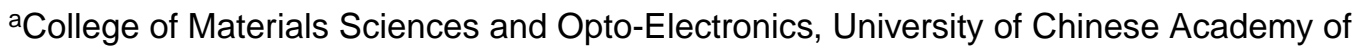 \\ Sciences, Beijing 100049 (China) \\ bepartment of Nanomedicine, Houston Methodist Research Institute, Houston, TX 77030 (USA) \\ 'Department of Biomedical Engineering, University of South Dakota, Sioux Falls, SD 57107 \\ (USA)
}

\section{Abstract}

A facile, one-step method was developed for in situ forming fluorescent silicon nanocrystals (SiNC) in microspherical encapsulating matrix. The obtained SiNC encapsulated polymeric microcapsules (SiPM) possess uniform size $(0.1-2.0 \mu \mathrm{m})$, strong fluorescence, and nanoporous structure. A unique two stage, time dependent reaction was developed, as the growth of SiNC was slower than the formation of polymeric microcapsules. The resulting SiPM with increasing reaction time exhibited two levels of stability, and in correspondence, the release of SiNC in aqueous media showed different behaviors. With reaction time $<1 \mathrm{~h}$, the obtained low-density SiPM (LD-SiPM) as matrix microcapsules, would release encapsulated SiNC on demand. With >1 $\mathrm{h}$ reaction time, resulting high-density SiPM (HD-SiPM) became stable SiNC reservoirs. SiPM exhibits stable photoluminescence. The porous structure and fluorescence quenching effects make SiPM suitable for bioimaging, drug loading and sorption of heavy metals $\left(\mathrm{Hg}^{2+}\right.$ as shown) with intrinsic indicator. SiPM was able to reduce metal ions, forming SiPM/Metal oxide and SiPM/ Metal hybrids, and their application in bio-sensing and catalysis were also demonstrated.

\section{Graphical Abstract}

Correspondence to: Xuewu Liu, xliu@houst onmethodist.org.

Electronic Supplementary Information (ESI) available: [details of any supplementary information available should be included here]. See DOI: $10.1039 / \mathrm{x} 0 \mathrm{xx} 00000 \mathrm{x}$ 


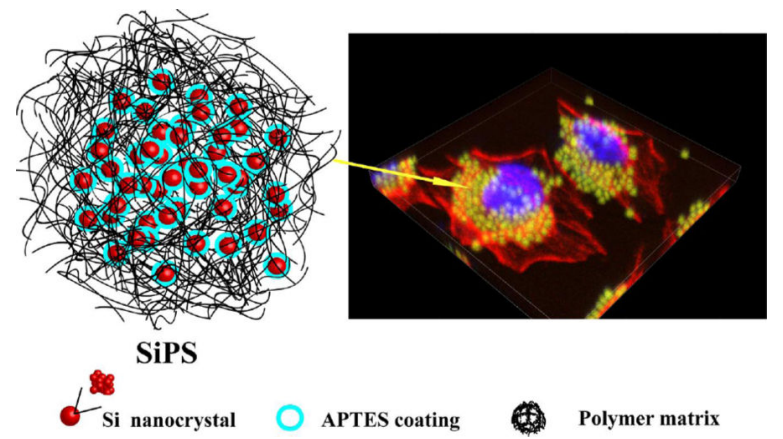

\section{Introduction}

Silicon-derived nanostructures (SiNC, ${ }^{1}$ nanowires, ${ }^{2}$ and porous silicon, ${ }^{3}$ etc.) have demonstrated excellent potential in the applications of photonics, ${ }^{4-6}$ electronics ${ }^{7-10}$ and biotechnology 11,12 owing to their unique photonic properties, ${ }^{13}$ biocompatibility, readily available surface chemistry ${ }^{14}$ and biodegradability. ${ }^{15}$ Recently, SiNC have attracted particularly research interests for their unique luminescence properties as result of quantum confinement effect. ${ }^{11}$ Compared to traditional fluorescence dyes and quantum dots, SiNC show better biocompatibility and fluorescence stability while eliminating toxic heavy metals contained in conventional quantum dots, which makes SiNC promising for both in vitro and in vivo long-term bioimaging. ${ }^{16}$ Various methods have been developed to produce SiNC, including reducing inverse micelle method, ${ }^{17}$ Zintl salts in inert organic solvents, ${ }^{18}$ reduction of silane precursors by sodium metal, ${ }^{19}$ laser pyrolysis of silane and plasma synthesis. ${ }^{20}$ Surface modification strategies were also studied to tailor SiNC's hydrophobicity and surface charge to enrich functionalities and extend biomedical applications. ${ }^{1}$ A few recent studies reported one-pot synthesis of amino modified SiNC by directly reducing aminosilanes. ${ }^{15,21}$

However, current applications of SiNC are quite limited. This is partially due to the constraints on size range of SiNC that can emit fluorescence. To achieve quantum confinement effect, the SiNC size has to be in a narrow range near Si excitation Bohr radius. ${ }^{11,22-24}$ Separation of small SiNC usually relies on costly dialysis process or ultrafiltration centrifugal devices. $^{25}$

Recent researches attempted to overcome these issues by cross-linkling SiNC into bigger nanoparticles, ${ }^{26}$ or assemble existing SiNC inside larger structures, such as mesoporous organosilica ${ }^{27}$ and polymers. ${ }^{28,} 29$ These hybrids integrated the properties of SiNC and matrix to achieve synergistic effects, thus could greatly extend their potential in areas such as fluorescence probe, optics, photonics, catalyst and energy. ${ }^{30}$ In addition, the supporting matrix also may form excellent SiNC storage to maintain robust photostability under harsh conditions. ${ }^{31}$ However, due to the complexity of current procedures used for assembling SiNC hybrids, very few studies have been reported, and the application of SiNC has been limited to imaging. A simple method to synthesize SiNC containing microcapsules that may release SiNC on demand is desired to extpand the field of SiNC applications. 
Here, for the first time, we reported a single-step, highly efficient approach to simultaneously synthesize fluorescent SiNC contained in polymeric microcapsules by controlling the reaction of aminosilane and L-ascorbic acid (L-AA). The reaction was carried out in alcohols at room temperature. While the resulting SiPM exhibit the typical fluorescence of SiNC, their sphere size $(0.1-2.0 \mu \mathrm{m})$, fluorescence intensity, porous structure and stability are tunable by controlling the reaction condition. Matrix microcapsules that will release SiNC in biological media can be synthesized. Taking the advantage of integrated properties of SiNC and porous structure of SiPM, we are able to develop a versatile platform for imaging, biosensing, catalysis, and removal of heavy metal contamination. The nanoporous structure and abundant amine groups make SiPM suitable in sorption of heavy metals $\left(\mathrm{Hg}^{2+}\right)$, and the fluorescence intensity of SiNC inside can be used as a probe to monitor the amounts of heavy metals $\left(\mathrm{Hg}^{2+}\right)$ in SiPM. We also demonstrate that porous SiPM can reduce metal ions, forming SiPM/Metal oxide $\left(\mathrm{SiPM} / \mathrm{MnO}_{2}\right)$ and $\mathrm{SiPM} / \mathrm{Metal}$ composites (for example, SiPM/Au and $\mathrm{SiPM} / \mathrm{Pt}$ ), which show potential in biosensing and catalysis.

\section{Results and discussion}

\section{Synthesis and characterization of SiPM}

SiPM were synthesized by controlling the reaction of aminosilanes with L-AA. A proposed scheme of SiPM synthesis is illustrated in Fig. 1A. In a typical preparation, (3aminopropyl)-trimethoxysilane (APTES) and L-AA (molar ratio [APTES]: [L-AA] $\approx 3: 1$ ) were used as starting materials to synthesize SiNC and polymer matrices. The reaction was carried out in isopropanol (IPA) containing a small portion $(\sim 5 \%)$ of water, which was essential for modulating the hydrolysis of APTES. A part of the APTES was hydrolyzed and condensed to form siloxane-like polymer, ${ }^{32-35}$ as illustrated in Supporting Information (SI) Equation 1, while another part of the APTES was reduced by L-AA to yield SiNC. ${ }^{36}$ L-AA attached on the siloxane-like polymer mainly by non-covalent bonding (electrostatic adherence, etc) or hydrogen bond, which accelerated the formation of polymeric matrices. As the matrices condensed further, APTES, L-AA, and SiNC crosslinked into a larger spherical structure, which became a porous scaffold for further in-situ growth of more SiNC. After this reaction, the SiPM were separated through centrifugation, and washed with IPA three times followed by storing in IPA.

Unlike reported methods for preparing SiNC that involve microwave heating, ${ }^{15}, 37,38 \mathrm{UV}$ irradiation ${ }^{21}$ or instant laser ablation, ${ }^{39}$ our one-step synthesis method can be carried out by simply mixing starting materials in alcohols at room temperature. Obtained SiNC embedded microcapsules have uniform sizes ranging between 0.1-2.0 $\mu \mathrm{m}$ (prepared so far). The yield can reach about $60 \%$ in terms of $\mathrm{Si}$ content from the staring APTES. As-prepared porous SiPM are positively charged, water dispersive, highly fluorescent, biocompatible and biodegradable.

Fig. 1B-E shows typically synthesized porous SiPM, encapsulated SiNC and induced fluorescence. As shown in scanning electron microscopy (SEM, Fig. 1B) and transmission electron microscopy (TEM, Fig. 1C inset) images, SiPM appear as uniform-sized spheres (1 $\mu \mathrm{m}$ shown, $<10 \%$ variation by estimation). High-resolution TEM (HRTEM, Fig. 1C and Fig. 
S1) images of SiPM (70 nm thin slices) show that SiNC ( $\sim 2 \mathrm{~nm}$ in diameter) are uniformly distributed whose morphology and size are similar with results shown in previous studies, and the lattice spacing of the crystal is $0.31 \mathrm{~nm}$, which matches the diamond lattice of $\mathrm{Si}$ (111). Elemental analyses of the SiPM (EDS, Fig. S2; XPS, Fig. S3) confirm the presence of $\mathrm{Si}, \mathrm{C}, \mathrm{N}$ and O. Both fresh SiPM and hydrolyzed SiPM are positively surface-charged (zeta potential, Fig. S4), most likely due to the presence of positively-charged amine group derived from APTES. This aminopropyl group becomes a side chain of polymer matrices after condensation. Similarly SiNC also show positive zeta potential (Fig. S4B) due to APTES surface coating. Positive charges are beneficial to keep SiPM dispersed in aqueous solutions. Fig. 1D shows absorption and photoluminescence spectra (PL) of SiPM. The broad absorption curve could be a result of light scattering of polymer matrices in SiPM. The as-prepared SiPM exhibit green fluorescence under UV irradiation. Inset shows captured photos of dispersed SiPM with and without UV irradiation at $365 \mathrm{~nm}$. Fig. 1E shows N2 adsorption/desorption isotherms of SiPM ( $24 \mathrm{~h}$ reaction time), showing a microporous structure. Pore-size distribution curve (inset) is obtained from the adsorption branch through Dubinin-Astakov (DA) method. ${ }^{40}$

\section{Formation process of SiPM}

In SiPM synthesis, formation of spherical polymer matrices and SiNC can be monitored through particle analysis and fluorescence measurement, respectively. A Beckman Multisizer 4 coulter counter was used to measure the size and concentration of SiPM (0.4$12 \mu \mathrm{m}$ detectable range with $20 \mu \mathrm{m}$ aperture). As shown in Fig. 2A and 2B, particle analysis has revealed a unique two-stage process. SiPM properties from two stages are distinctive. In the first stage (from beginning to about $1 \mathrm{~h}$ ), both mean sphere size and amounts (sphere $>0.4 \mu \mathrm{m}$ ) increase rapidly. Then both rates drastically drop by $\sim 40$ folds (calculated by comparing the slope of curves of each stage) after 1st $\mathrm{h}$ (starting second stage). During the second stage, there is no obvious change of size and amounts of SiPM. This drastic drop is likely due to the significant consumption of starting materials and the existence of critical concentration. However, if we change the concentration of starting APTES and L-AA, the size and yield of final SiPM are correspondingly affected. As seen in Fig. S5, SiPM with size range $0.4 \sim 2.0 \mu \mathrm{m}$ were successfully synthetized by varying APTES concentration. By using other aminesilanes (such as $\mathrm{N}$-(2-aminoethyl)-3-(trimethoxysilyl)propylamine or 2-[2(3-trimethoxysilylpropylamino)ethylamino]ethylamine) instead of APTES, SiPM as small as $0.1 \mu \mathrm{m}$ had been obtained. The photoluminescence intensity of SiPM at $500 \mathrm{~nm}$ (excitation wavelength: $420 \mathrm{~nm}$ ) also increased with reaction time (Fig. S6). However, unlike the trends observed for sphere size and amounts, the fluorescence intensity increased quite linearly in the first $2 \mathrm{~h}$, then changed to a standard exponential curve of first-order reaction (Fig. 2C). This means SiNC formation process saturates slower than the sphere formation, since fluorescence intensity represents the quantity of encapsulated SiNC. A possible explanation is that SiNC continue to be reductively formed in pores. Even though spherical porous matrices are formed in $1 \mathrm{~h}$, it takes longer for SiNC to fill the pores.

In the first stage, formation of siloxane-like polymer matrices is very fast and SiNC embedding is much slower, which results a highly-porous, low density SiPM (LD-SiPM). Then the sphere formation slows down significantly to maintain a critical size and 
concentration, while the SiNC continue to be formed in the polymer matrices. Finally less porous, high density SiPM (HD-SiPM) were obtained. As shown in Fig. 2D and 2E, LDSiPM have a smooth appearance, while HD-SiPM show nano-topographic structure possibly due to clustered surface SiNC. The increase in hardness along with time is also verified via force-displacement curves (shown in Fig. 2F). Moreover, the solution color of HD-SiPM is darker than LD-SiPM (Fig. S7). The elemental composition (Table S1) also demonstrates increasing ratio of $\mathrm{Si}$ in $\mathrm{HD}-\mathrm{SiPM}$. All this data indicates that $\mathrm{SiNC}$ continue to form in second stage of the reaction with time. More detailed SEM analysis of SiPM (Fig. 3) shows that soft siloxane-like polymer matrices are formed in the first $10 \mathrm{~min}$, while during next 10 min matrices are changed from aggregated state to uniformly-sized spheres, Then, the color of solution changes from ivory-white to light-yellow (without UV irradiation, shown in Fig. S8), which indicates SiNC embedding starts later than matrices formation HD- and LDSiPM exhibit distinctive porous structure. BET analyses of LD-SiPM after $30 \mathrm{~min}$ of reaction time and HD-SiPM after $24 \mathrm{~h}$ of reaction time (Fig. S9 and Fig. 1E) show mesoporous $(\sim 2.8 \mathrm{~nm})$ and microporous $(\sim 0.9 \mathrm{~nm})$ structure, respectively, indicating SiNC packing associated with reduced pore size in SiPM. As physical absorption specificity is heavily determined by the porous structures, two distinctive porous SiPM may perform differently in molecular absorption, which is under investigation.

\section{SiNC releasing from SiPM}

LD- and HD-SiPM are distinctive in hydrolytic degradability. The hydrolysis curves of both SiPM were calculated through measuring PL spectra of released SiNC from microcapsules in DI water and PBS, and shown in Fig. 4A. Clearly, SiNC were released faster in PBS, cell culture medium than $\mathrm{H} 2 \mathrm{O}$. In fact, the structure of LD-SiPM (reaction time $30 \mathrm{~min}$ ) collapsed in $2 \mathrm{~h}$, as $100 \%$ SiNC were released. However, HD-SiPM shows higher stability, with less than $30 \%$ SiNC clustered nanoparticles (SiNP) falling off from SiPM. This is likely due to more complete cross-linking, denser SiNC packing, and smaller pore size. Fig. 4B shows residual of LD-SiPM after $24 \mathrm{~h}$ soaking in $\mathrm{H}_{2} \mathrm{O}$. Many $\operatorname{SiNP}(<100 \mathrm{~nm})$ are found from collapsed structure of microcapsules, which indicates unstable crosslinking of SiNP and siloxanes in capsules. The LD-SiPM dispensed solution become clear (Fig. 4C) in $24 \mathrm{~h}$. LD-SiPM are able to be hydrolyzed in PBS as soon as in a few hours, depending on the reaction time (as seen in Fig. S10, the hydrolysis time-reaction time curve). However, there is no obvious change for HD-SiPM. In order to quantify the hydrolytic behavior of HDSiPM, Mutisizer was used to quantify the size and amounts of HD-SiPM during hydrolysis. Fig. 4D shows there is decreasing trend for diameter of HD-SiPM but without a corresponding decrease in particle amounts. There are much less SiNP fall off from HDSiPM (Fig. 4E, inset SEM images and Fig. S11), which is also in accordance with Mutisizer results (The diameter of HD-SiPM changes from $1.6 \mu \mathrm{m}$ to $1.39 \mu \mathrm{m}$ ) and released curve measured by ICP-OES (Fig. S12).

Compared with free-standing SiNC of a few nanometers, SiPM has several advantages due to their size. First, they are easy to handle. They can be collected, processed and stored through standard centrifugation and filtration processes. The microcapsule (LD-SiPM) can be used for SiNC storage, where SiNC can be released on demand. Second, nanopores in SiPM provide structural basis for absorption of molecules, making it possible for chemical 
detection, ${ }^{41}$ catalysis, ${ }^{42}$ drug delivery and controlled release. With their fluorescence, SiPM are also promising as fluorescent probes. Integration of these functionalities opens broad opportunities, deserving further investigations. We explored the capability of SiPM to adsorb and reduce metal ions in aqueous solution, and confirmed that SiPM were able to reduce noble metal ions to generate metal oxides and metal nanoparticles in SiPM, which also indicates the existence of L-AA in SiPM. This also altered the fluorescence of SiPM. Resulting SiPM-metal hybrid particles may have potential applications in catalysis ${ }^{43}$ and sensing. ${ }^{44}$

\section{SiPM as a photostable fluorescent drug delivery vector}

It is worth noting that gel-like siloxane-derived hybrids have also been reported with comparable fluorescence properties. ${ }^{33,} 34,45$ However, special treatments such as calcination ${ }^{46,47}$ and instant ablation ${ }^{39}$ are required for the synthesis. Based on our experiments, APTES in hydrochloric acid solutions formed ivory-white hybrid, but did not show fluorescent property (data not shown). According to previous reports, carboxylic acids are critical by forming $\mathrm{C}$ substitutional defect for $\mathrm{Si}$, to induce fluorescence in APTES-based hybrids. ${ }^{48}$ These treatments are neither available nor needed for SiPM to trick the fluorescence. Moreover, SiPM lose their fluorescence if calcinated in air, implying the effect of SiNC oxidation (data not shown). On the other hand, the emission spectrum of hydrolyzed products of SiPM (mainly composed of SiNC) is similar to that before hydrolysis. These suggest the embedded SiNC (rather than the polymer matrices) to be the source of fluorescence of SiPM.

Besides quantum size effect of SiNC, the surface effects may also have an impact on their fluorescence. ${ }^{11}$ The surface-capping molecules such as allylamine ${ }^{49}$ and APTES ${ }^{15}$ play an important role in the radiative combination mechanisms in $1-2 \mathrm{~nm} \mathrm{SiNC},{ }^{50}$ since their electronic charge distribution can be modified by the polar nature of their capping ligands. Therefore, the size and amine group on SiNC in SiPM are responsible for their fluorescent emission SiPM exhibits strong fluorescence, and the quantum yield (QY) of released SiNC is similar to that of free $\mathrm{SiNC}$ (QY: $\sim 20 \%$, PL spectrum of quinine sulfate, as a reference, shown in Fig. S13) prepared before, ${ }^{36}$ and siloxane-like matrices do not seem to quench the fluorescent emission, making SiPM attractive for bio-imaging. The photostability of SiPM and free SiNC was also evaluated under UV irradiation. As shown in Fig. 5A, the PL intensities of both samples continuously decreased along with illumination time. SiNC shows a much faster decay rate than SiPM, and the remained PL intensity of SiNC decreased to $25 \%$ while the SiPM maintained a higher PL intensity value of $62 \%$. We assessed submicron SiPM's fluorescence performance and biocompatibility for cell imaging. The cell uptake and intracellular behavior of SiPM by Hela cells were also explored by monitoring green fluorescence (ex. $488 \mathrm{~nm}$, em. 500-550 nm). To co-localize particles, DAPI and Alexa fluor 555 Phallodin were used for staining nuclei and actin respectively. The particles eventually accumulate in cytoplasm near cell nuclear envelopes after $4 \mathrm{~h}$ (Fig. 5B). In addition, nanopores in SiPM provides structural basis for absorption of molecules, making it possible for chemical detection, ${ }^{41}$ catalysis, ${ }^{42}$ drug delivery and controlled release. With their porosity, SiPM also are promising as bi-functional delivery vectors (for imaging and drug delivery). ${ }^{51}$ We tested the loading of doxorubicin in SiPM, and corresponding loading 
capacity is shown in Fig. S14. SiPM demonstrates high biocompatibility with Hela cells with over $95 \%$ viable cells after $24 \mathrm{~h}$, even with very high particle concentration (> 1 million/mL SiPM) (Fig. 5C). When cells are incubated with SiPM/DOX and free DOX, concentration-dependent cell death is observed. Higher concentration of DOX would result in decrease of cell viability. The integration of these functionalities with fluorescence property opens broad opportunities, deserving further investigations.

\section{SiPM/ $/ \mathrm{MnO}_{2}$ hybrids for glutathione (GSH) detection}

Glutathione ( $\gamma$-L-glutamyl-L-cysteinyl-glycine; GSH), as the main non-protein thiol source, exists in most mammalian tissues. ${ }^{52}$ It is necessary to monitor level of glutathione due to its biological significance in diseaces such as cancer, ${ }^{53} \mathrm{HIV}^{54}$ and heart problems. ${ }^{55} \mathrm{SiPM}$ were able to in situ reduce $\mathrm{KMnO}_{4}$ to $\mathrm{MnO}_{2}$ sheets by immersing SiPM in $\mathrm{KMnO}_{4}$ aqueous solutions (here we used HD-SiPM). Formed $\mathrm{MnO}_{2}$ sheets were able to quench the photoluminescence of SiPM, which is recoverable when GSH was added. ${ }^{56}$ The relationship between $\mathrm{KMnO}_{4}$ amounts and quenching efficiency of formed $\mathrm{MnO}_{2}$ on $\mathrm{SiPM}$ was shown in Fig. 6A and 6B. Inset in Fig. 6B is a photo of corresponding $\mathrm{SiPM} / \mathrm{MnO}_{2}$ solutions. $\mathrm{MnO}_{2}$ formed by using $2 \mathrm{mM} \mathrm{KMnO}_{4}$ in reaction reached about $98 \%$ quenching efficiency on SiPM. The morphology of SiPM/ $\mathrm{MnO}_{2}$ slices (thickness: $70 \mathrm{~nm}$ ) is observed through TEM (Fig. 6C). The morphology of SiPM is not significantly changed but on enhanced contrast. The existence of $\mathrm{Mn}$ on $\mathrm{SiPM} / \mathrm{MnO}_{2}$ is also verified by EDS (Fig. S15). The recovery of photoluminescence is realized through red-ox reaction between $\mathrm{MnO}_{2}$ in $\mathrm{SiPM}$ and GSH, and the fluorescent spectra of this process is shown in Fig. 6D. The amount of photoluminescence that is recovered is proportional to the GSH concentration. It is noted that there is hardly fluorescence recovery for control sample in Fig. 6D compared with other samples (with GSH addition). Therefore, SiNC releasing causes little influence on final results. The limit of detection (LOD) LOD is adjustable by changing amounts of $\mathrm{KMnO}_{4}$ in reaction and $\mathrm{SiPM} / \mathrm{MnO}_{2}$ in detection.

\section{HD-SiPM applied in $\mathrm{Hg}^{2+}$ sorption}

Mercury is one of the poisonous heavy metals where the residual level of its ionized form $\left(\mathrm{Hg}^{2+}\right)$ in drinking water remains a big problem for environmental science and pollution research. ${ }^{57}$ Though amine terminated SiNC had been used as fluorescent probe in detecting $\mathrm{Hg}^{2+}$ in aqueous solutions via the affinity of $\mathrm{Hg}^{2+}$ to $\mathrm{N},{ }^{58}$ previously used $\mathrm{SiNC}$ is dissociated, lacking the capability to deplete $\mathrm{Hg}^{2+}$. On contrary, the highly nanoporous structure in our SiPM is suitable in adsorbing molecules. A detection-sorption multiplexed platform of $\mathrm{Hg}^{2+}$ was developed by integrating porous structure and fluorescence of SiPM. The adsorption curve of $\mathrm{Hg}^{2+}$ at different concentrations by using SiPM is shown in Fig. 7A. HD-SiPM shows high adsorption capacity, more than $300 \mathrm{mg} \mathrm{Hg}^{2+}$ can be adsorbed in $1 \mathrm{~g}$ $\mathrm{SiPM}$. Also the adsorption process is highly efficiency, usually saturated within $1 \mathrm{~min}$, thus the small release of SiNC hardly affect detection accuracy. The relative fluorescent quenching efficiency of $\mathrm{Hg}^{2+}$ on SiPM is shown in Fig. 7B. $10^{-3} \mathrm{M} \mathrm{Hg}^{2+}$ shows about $50 \%$ fluorescent quenching on photoluminescence spectrum of SiPM, which, however, cab be recovered with GSH addition $\left(10^{-3} \mathrm{M}\right)$. The nanoporous structure of SiPM is responsible for adsorbing $\mathrm{Hg}^{2+}$, while SiNC in SiPM performs as fluorescent probe for monitoring adsorbed 
$\mathrm{Hg}^{2+}$. The detection-sorption multiplexed platforms are recyclable simply by using GSH to recover $\mathrm{Hg}^{2+}$ from the SiPM.

\section{SiPM/Au/Pt composites for catalyzing 4-Nitrophenol (4-NP)}

The SiPM were proven to reduce noble metal ions into nanoparticles from their aqueous solutions due to the existence of L-AA in SiPM. In our SiPM, we show that reduction can happen in situ at the nanopores. Here we demonstrate that the resulting silicon-metal nanocomposite can be used for catalysis of redox reaction. Fig. 8A shows TEM image of $\mathrm{SiPM} / \mathrm{Au}$. The distribution of Au nanoparticles (AuNP) in SiPM seems to match the porous structure in SiPM. AuNP are mostly seen on outer surface, and there is a blank shell portion where no AuNP are visible. There are also AuNP visible at center, indicating SiNC are also nucleated deeply in SiPM through diffusion of ions. Fig. 8B, Fig. S16 and Fig. S17 show EDS spectra of SiPM/Au $0.5 / \mathrm{Pt}_{0.5}$, SiPM/Au and SiPM/Pt (HD-SiPM were applied). The reduction of 4-nitrophenol (4-NP) to 4-aminophenol (4-AP) by NaBH4 was used to examine the catalytic activity of SiPM/metal composites. ${ }^{59} \mathrm{Fig}$. $8 \mathrm{C}$ shows catalytic conversion of 4 $\mathrm{NP}$ by SiPM/ $/ \mathrm{Au}_{0.5} \mathrm{Pt}_{0.5}$, inset is the principle of reduction. The absorption peak of 4-NP was at $317 \mathrm{~nm}$ and red-shifted to $400 \mathrm{~nm}$ after $\mathrm{NaBH}_{4}$ has been added, due to ionization of 4-NP. When the SiPM/metal composites were added, the color of the reacting solution changed from bright yellow to colorless, which reflects the diminishing absorbtion at $400 \mathrm{~nm}$. The reaction rate can be evaluated via slope of kinetic curves since concentration of $\mathrm{NaBH}_{4}$ was excessive compared with that of 4-NP. Fig. 8D shows change of $\ln (\mathrm{C} / \mathrm{Co})$ along with reaction time. The plots can be linearly fitted by the equation: $\ln (\mathrm{C} / \mathrm{Co})=\mathrm{kt} . \mathrm{C}$ and $\mathrm{Co}$ stand for reactive and initial absorbance intensity at $400 \mathrm{~nm}$. $\mathrm{k}$ stands for the rate constant, which can be used to calculate catalytic ability of these composites. ${ }^{59} \mathrm{SiPM} / \mathrm{Au}_{\mathbf{0 . 5}} \mathrm{Pt}_{0.5}$ $(\mathrm{k}=0.492)$ demonstrate the highest catalytic ability compared to $\mathrm{SiPM} / \mathrm{Au}(\mathrm{k}=0.162)$ and $\mathrm{SiPM} / \mathrm{Pt}(\mathrm{k}=0.168)$. Reducing metal ions $(\mathrm{Cu}, \mathrm{Pt}, \mathrm{Ag}$ and $\mathrm{Au}$ verified) to form $\mathrm{SiPM} /$ metal composites greatly extends the potential application of SiNC in applications such as detection, antibiosis, Surface Enhanced Raman Scattering (SERS) and drug delivery.

\section{Chemical composition analysis of SiPM}

The organic composition of SiPM is mostly APTES derived siloxane-like polymer (Equation S1). The chemical structures are confirmed by the diverse spectroscopies. The FTIR (Fig. $\mathrm{S} 18$ ) exhibits characteristic peaks of Si-O-Si from siloxane-like backbone, C-H stretching, $\mathrm{O}-\mathrm{H}$ stretching, stretching and bending of $\mathrm{N}-\mathrm{H}$ bond, and potential $\mathrm{C}=\mathrm{C}$ and $\mathrm{C}=\mathrm{O}$ stretching vibrations, suggesting the existence of L-AA in polymeric matrices. NMR spectrum further confirms the existence of both APTES-derived siloxane-like polymer and L-AA (Fig. 9A), the peaks at 2.9, 1.7 and $0.6 \mathrm{ppm}$ are corresponding to Propyl group in APTES, and it is significant to note the loss of up to three ethoxy groups for APTES during coupling of molecules in forming polymer matrices and to surfaces of silicon nanoparticles. ${ }^{49,}{ }^{60}$ For measuring NMR spectrum of AA, " $\mathrm{D}_{2} \mathrm{O}$ shake" occurs when using $\mathrm{D}_{2} \mathrm{O}$ as solvent in NMR testing, in which the exchangeable protons are exchanged with the deuterium in $\mathrm{D}_{2} \mathrm{O}$ and disappear in spectrum. ${ }^{61}$ The pattern at 3.7 and 3.9 ppm are distinguished as $-\mathrm{CH}_{2}$ group linked with hydroxyl in AA. The signal at $4.4 \mathrm{ppm}$ is attributed to the proton on the heterocycle. A sharp peak observed at $1.1 \mathrm{ppm}$ is due to the residue of IPA molecules in samples. In 2D-TOCSY spectrum (shown in Fig. S19), one set shows typical correlation and 
chemical shift of propyl group in APTES, while the other set matches well to that of L-AA. The resonance of former set is roughly two times stronger than the latter one, suggesting less abundance of L-AA in matrices. This is consistent with elemental analysis and zeta potential measurements. There are two facts worthy of noticing. First, no protons from ethyloxy groups are observed, suggesting APTES is mostly hydrolyzed during condensation. ${ }^{49,} 60$ Second, neither short- nor long-range coupling is observed between APTES-derived siloxane and L-AA. This suggests that the incorporation of L-AA in the siloxane-like backbone could be through non-covalent bonding (electrostatic adherence etc.) or hydrogen bond. The high resolution XPS spectra (Fig. S3 and Fig. 9B) indicates chemical bonds of C$\mathrm{C}, \mathrm{C}-\mathrm{N}, \mathrm{C}-\mathrm{O}, \mathrm{C}=\mathrm{O}$ and $\mathrm{Si}-\mathrm{O}$ in SiPM. While the existence of $\mathrm{C}-\mathrm{C}, \mathrm{C}-\mathrm{N}$ and $\mathrm{NH} 2$ is attributed to the aminopropyl side chain, the $\mathrm{Si}-\mathrm{O}$ is attributed to siloxane-like backbone. Fig. 9C shows UV absorbance curve of hydrolytic SiPM as compared to control of L-AA. (Note: The peak at around $350 \mathrm{~nm}$ is attributed to SiNC.) Both curves have a consistent sharp absorption at around $300 \mathrm{~nm}$, suggesting L-AA is preserved in polymer matrices.

\section{Conclusions}

In summary, we developed a one-step method for facile, highly efficient synthesis of SiNC contained in microcapsules. The as-prepared SiPM show uniform morphology, great aqueous dispersibility, strong photoluminescence, and porous structure. The size, yield, porosity, fluorescence intensity, and stability of SiPM are adjustable by simply changing reaction parameters. Combination of fluorescent $\mathrm{SiNC}$ and highly porous polymeric matrix makes SiPM an excellent self-indicator in adsorption $\mathrm{Hg}^{2+}$ in aqueous solution. SiPM can also reduce metal ions in their aqueous solution and alter their fluorescence. The formed $\mathrm{SiPM} / \mathrm{MnO}_{2}$ and $\mathrm{SiPM} / \mathrm{Au}_{0.5} / \mathrm{Pt}_{0.5}$ shows excellent performance in detecting GSH and catalytic activity of 4-NP. These SiPM/metal oxide and SiPM/metal nanoparticles can be applied in areas such as cancer therapy, ${ }^{62}$ biosensors ${ }^{44}$ and catalysis. ${ }^{43}$

\section{Experimental}

Materials

(3-Aminopropyl)-trimethoxysilane (APTES, 99\%), L-Ascorbic acid (L-AA, 99\%), Isopropanol (IPA), hydrogen tetrachloroaurate (III) hydrate $\left(\mathrm{HAuCl}_{4}\right)$, silver nitrate $\left(\mathrm{AgNO}_{3}\right)$, potassium permanganate $\left(\mathrm{KMnO}_{4}\right)$, mercury (II) chloride and quinine sulfate were purchased from Sigma-Aldrich. All chemicals were used without additional purification.

\section{Synthesis and characterization of fluorescent SiPM}

Silicon nanocrystals (SiNC) containing polymer microcapsules (SiPM) were synthesized by controlling the reaction of APTES and L-AA. Typically, $100 \mu \mathrm{L}$ APTES and $1.5 \mathrm{~mL}$ of 0.1 M L-AA aqueous solutions were added into $30 \mathrm{~mL}$ IPA. The mixture was briefly sonicated, and then kept at a specific temperature for defined time. Typical fluorescent SiPM with $\sim 1$ $\mu \mathrm{m}$ diameters were then obtained. After reaction, the SiPM were first precipitated through centrifugation at $10,000 \times \mathrm{g}$ for $5 \mathrm{~min}$, washed three times using IPA and then stored in IPA. Scanning Electron Microscopy (SEM, high vacuum, HV=8 kV, spot size 3.5), JEM-2100 Field Emission Gun Transmission Electron Microscope (TEM), X-ray Photoelectron (XPS) 
and EDS were utilized for characterizing the structure, morphology and elements of the prepared SiPM. Surface chemical band was monitored by Fourier Transform Infrared Spectroscopy (FTIR) and XPS. Malvern Zetasizer was used to assess zeta potential of SiPM. The composition of polymer matrix was tested by 1D 1H NMR and 2D TOCSY NMR. $\mathrm{SiPM}$ (reaction time, $20 \mathrm{~min}$ ) were hydrolyzed in $\mathrm{D}_{2} \mathrm{O}$. The hydrolytic sample was added into NMR tube and measured by $600 \mathrm{MHz}$ NMR.

\section{Tuning the size, yield, fluorescent intensity, porosity and biodegradability/hydrolysis of SiPM}

The influence of reaction time on the size of SiPM was explored. Briefly, after mixing APTES with L-AA in IPA, the mixture was allowed to react for various times (from $30 \mathrm{~min}$ to $24 \mathrm{~h}$ ), then the reaction was terminated by washing. In order to test the effect of reagent concentration on the final size of SiPM, $100 \mu \mathrm{L}$ APTES and $1.5 \mathrm{~mL} 0.1 \mathrm{M} \mathrm{L}-\mathrm{AA}$ aqueous solutions were added in IPA of different volumes (from $15 \mathrm{~mL}$ to $180 \mathrm{~mL}$ ). Multisizer 4 Coulter Counter (Beckman Coulter) was used to monitor the change of size and accounts of as-prepared SiPM. The impact of reaction time on fluorescent intensity of SiPM was investigated by measuring their photoluminescence curves by Synergy H4 Hybrid Reader (Bio TEK). $\mathrm{N}^{2}$ adsorption/desorption isotherms of different samples were measured using Quantachrome Automated Gas Sorption Analyzer, and the surface area and pore size distribution were calculated by Dubinin-Astakov (DA) and Barrett-Joyner-Halenda (BJH) method

In order to evaluate the SiNP release behavior from LD-SiPM and HD-SiPM, one billion SiPM are immersed in 500ul DI water and PBS respectively. The dispensed solutions were centrifuged at different time points, and the PL spectra of supernatant (SiNP) was recorded via plate reader. The precipitate was tested by SEM and Multisizer to monitor their morphology, size and amounts.

To test biodegradation kinetics of $\mathrm{Si}, 1.25 \times 10^{10} \mathrm{SiPM}(\mathrm{n}=3$ ) were dispersed into $500 \mu \mathrm{L}$ Phosphate Buffered Saline (PBS, pH=7.4). The mixtures were kept in the Digital Heatblock at $37{ }^{\circ} \mathrm{C}$. At different time points, $10 \mu \mathrm{L}$ SiPM solution was taken out and centrifuged, and the precipitate was extensively washed by IPA and centrifuged at $13,000 \times \mathrm{g}$ for three times, then spot on SEM stub for imaging their morphology change. At the same time point, SiPM were spun down, and supernatant was replaced with $500 \mu \mathrm{L}$ fresh PBS. All the supernatant was collected and diluted in DI water, and stored at $4{ }^{\circ} \mathrm{C}$ for following Si element analysis, which was measured by Inductively Coupled Plasma Optical Emission Spectrometer (ICPOES, Varian 720-ES).

SiNC are produced according previous method. ${ }^{36}$ Briefly, $100 \mu \mathrm{L}$ APTES is mixed with 1 $\mathrm{mL}$ of $0.1 \mathrm{M} \mathrm{L}-\mathrm{AA}$ and react for $1 \mathrm{~h}$. The residual reagents were removed by dialysis ( 1 $\mathrm{kDa}$ ). The resulting SiNC exhibit green fluorescence under UV irradiation.

The quatum yield of SiNC and release SiNC from SiPM were determined by using quinine sulfate as a standard, and QY values are based on the established equation: $\mathrm{Qx}=$ $\mathrm{Q}_{\mathrm{r}} \mathrm{M}_{\mathrm{x}} \mathrm{N}_{\mathrm{x}}{ }^{2} /\left(\mathrm{M}_{\mathrm{r}} \mathrm{N}_{\mathrm{r}}{ }^{2}\right) .{ }^{21,63} \mathrm{~N}, \mathrm{M}$, and $\mathrm{Q}$ stand for the average refractive index of the solvent, the gradient of straight line, and quantum yield. $\mathrm{r}$ and $\mathrm{x}$ indicate the reference quinine sulfate 
and the tested SiNC, respectively. The value of optical densities at the excitation wavelength of samples were adjusted to the same values $(\unlhd 0.1)$.

\section{CCK-8 assay and cell uptake of SiPM}

The Human cervical cells Hela were cultured in Dulbecco's modified Eagle's medium (DMEM) supplemented with $10 \%$ fetal bovine serum (FBS) and antibiotics $(100 \mu \mathrm{g} / \mathrm{mL}$ streptomycin and $100 \mathrm{U} / \mathrm{mL}$ penicillin). Both cell lines were cultured under the condition of $5 \% \mathrm{CO}_{2}$ and $95 \%$ humidity at $37{ }^{\circ} \mathrm{C}$.

Suspensions of $1 \times 10^{5}$ cells $/ \mathrm{mL}$ Hela were plated in Lab-Tek Chamber Slides (Thermo Fisher Scientific, USA) and cultured overnight, followed by adding SiPM $\left(2.5 \times 10^{6} / \mathrm{mL}\right)$. They were cultured for $1 \mathrm{~h}$ and $4 \mathrm{~h}$ separately. After washed by PBS for 3 times, the cells were fixed with $4 \%$ glutaraldehyde in PBS for $30 \mathrm{~min}$ at room temperature. The fixed cells were stained with DAPI before observing by Nikon A1 Confocal Imaging System. Counting Kit-8 (CCK-8) assay was applied to evaluate cytotoxicity. Briefly, $1 \times 10^{4}$ Hela cells were plated separately in 96-well plates and cultured overnight. Then they were exposed to SiPM, $\mathrm{SiPM} / \mathrm{DOX}$ and free DOX with various concentrations for $24 \mathrm{~h}$. After $4 \mathrm{~h}$ of $10 \mu \mathrm{L} \mathrm{CCK}-8$ addition, the absorption peak of medium at $450 \mathrm{~nm}$ and $630 \mathrm{~nm}$ by Synergy H4 Hybrid Reader (Bio TEK).

\section{Synthesis of SiPM/MnO ${ }_{2}$ for detecting glutathione (GSH) in aqueous solutions}

$\mathrm{SiPM} / \mathrm{MnO}_{2}$ composites were synthetized simply by mixing $\mathrm{SiPM}$ with $\mathrm{KMnO}_{4}$ aqueous solutions. Briefly, various volumes of $40 \mathrm{mM} \mathrm{KMnO}_{4}$ was added in SiPM dispensed aqueous solution (with about $1 \times 10^{8} \mathrm{SiPM}$ ), and reacted for $2 \mathrm{~h}$. After reaction, the formed $\mathrm{SiPM} / \mathrm{MnO}_{2}$ was collected via centrifugation at $10,000 \times \mathrm{g}$ for $5 \mathrm{~min}$, washed by IPA for three times and then stored in IPA. In order to test GSH detection using SiPM/ $\mathrm{MnO}_{2}$ composites. GSH solutions at different concentrations were added in $\mathrm{SiPM} / \mathrm{MnO}_{2}$ composites solutions. The change of photoluminescence spectra were recorded by plate reader.

\section{SiPM applied in $\mathrm{Hg}^{2+}$ adsorption}

The stock solution of $1000 \mathrm{ppm}$ of $\mathrm{Hg}^{2+}$ was prepared by dissolving $13.54 \mathrm{mg} \mathrm{HgCl}_{2}$ in 10 mL DI water. $10 \mu \mathrm{L}$ of $1 \mathrm{~B} / \mathrm{mL}$ HD-SiPM were mixed with $\mathrm{Hg}^{2+}$ at different concentrations. The PL spectra of SiPM/ $\mathrm{Hg}^{2+}$ were recorded by plate reader. After $5 \mathrm{~min}$, the supernatant was collected via centrifugation and measured by ICP-OES to calculate Hg amounts inside.

\section{Synthesis of SiPM/Au, SiPM/Pt and SiPM/Au0.5Pt0.5 for catalytic activity on 4-Ntrophenol}

For SiPM/Au, briefly, $30 \mu \mathrm{L}$ of $20 \mathrm{mM} \mathrm{HAuCl}_{4}$ was added in SiPM dispensed aqueous solution (with about $1 \times 10^{8} \mathrm{SiPM}$ ), and reacted for $24 \mathrm{~h} .2 \mu \mathrm{L}$ of $300 \mathrm{mM} \mathrm{K}_{2} \mathrm{PtCl}_{4}$ was reacted with SiPM in forming SiPM/Pt. For SiPM/ $/ \mathrm{Au}_{0.5} \mathrm{Pt}_{0.5}, 15 \mu \mathrm{L}$ of $20 \mathrm{mM} \mathrm{HAuCl} 4$ and $2 \mu \mathrm{L}$ of $300 \mathrm{mM} \mathrm{K}_{2} \mathrm{PtCl}_{4}$ were simultaneously mixed with SiPM solutions. After reaction, the formed composites were collected via centrifugation at $10,000 \times \mathrm{g}$ for $5 \mathrm{~min}$, washed by IPA for three times and then stored in IPA. 
The reduction of 4-nitrophenol (4-NP) by NaBH4 was used to test the catalytic activity of $\mathrm{SiPM} /$ metal composites in aqueous solutions. Briefly, $2 \mu \mathrm{L}$ of $0.1 \mathrm{M}$ 4-NP and $10 \mu \mathrm{L}$ of $1 \mathrm{M}$ $\mathrm{NaBH} 4$ were added in $2 \mathrm{~mL}$ DI water in cuvette. Then $80 \mu \mathrm{L}$ of SiPM/metal (SiPM/Au, $\mathrm{SiPM} / \mathrm{Pt}$ and SiPM/Au0.5Pt0.5) composites were added in the cuvette. The UV-vis spectrometer was used to record the change of absorbance curves along with time.

\section{Supplementary Material}

Refer to Web version on PubMed Central for supplementary material.

\section{Acknowledgments}

The authors would like to thank Dr. Jianhua Gu of the HMRI Microscopy-SEM/AFM core on SEM imaging and Mr. Carlos Favela for his help on Confocal imaging. The authors acknowledge financial support from the following sources: NIH U54CA151668-01, NIH 1R21CA190024-01, DOD W81XWH-12-1-0414, DOD W81XWH-10-2-0125, National Natural Science Foundation of China 81571791, and Houston Methodist Research Institute.

\section{Notes and references}

1. Cheng XY, Lowe SB, Reece PJ, Gooding JJ. Chemical Society Reviews. 2014; 43:2680-2700. [PubMed: 24395024]

2. Wang YL, Wang TY, Da PM, Xu M, Wu H, Zheng GF. Advanced Materials. 2013; 25:5177-5195. [PubMed: 23828226]

3. Park JH, Gu L, von Maltzahn G, Ruoslahti E, Bhatia SN, Sailor MJ. Nature Materials. 2009; 8:331336. [PubMed: 19234444]

4. Almeida VR, Barrios CA, Panepucci RR, Lipson M. Nature. 2004; 431:1081-1084. [PubMed: 15510144]

5. Liu AS, Jones R, Liao L, Samara-Rubio D, Rubin D, Cohen O, Nicolaescu R, Paniccia M. Nature. 2004; 427:615-618. [PubMed: 14961115]

6. Baehr-Jones T, Pinguet T, Lo Guo-Qiang P, Danziger S, Prather D, Hochberg M. Nature Photonics. 2012; 6:206-208.

7. Staude I, Miroshnichenko AE, Decker M, Fofang NT, Liu S, Gonzales E, Dominguez J, Luk TS, Neshev DN, Brener I, Kivshar Y. Acs Nano. 2013; 7:7824-7832. [PubMed: 23952969]

8. Zwanenburg FA, Dzurak AS, Morello A, Simmons MY, Hollenberg LCL, Klimeck G, Rogge S, Coppersmith SN, Eriksson MA. Reviews of Modern Physics. 2013; 85:961-1019.

9. Hwang SW, Tao H, Kim DH, Cheng HY, Song JK, Rill E, Brenckle MA, Panilaitis B, Won SM, Kim YS, Song YM, Yu KJ, Ameen A, Li R, Su YW, Yang MM, Kaplan DL, Zakin MR, Slepian MJ, Huang YG, Omenetto FG, Rogers JA. Science. 2012; 337:1640-1644. [PubMed: 23019646]

10. Wu H, Chan G, Choi JW, Ryu I, Yao Y, McDowell MT, Lee SW, Jackson A, Yang Y, Hu LB, Cui Y. Nature Nanotechnology. 2012; 7:309-314.

11. McVey BFP, Tilley RD. Accounts of Chemical Research. 2014; 47:3045-3051. [PubMed: 25252604]

12. Tasciotti E, Liu XW, Bhavane R, Plant K, Leonard AD, Price BK, Cheng MMC, Decuzzi P, Tour JM, Robertson F, Ferrari M. Nature Nanotechnology. 2008; 3:151-157.

13. Priolo F, Gregorkiewicz T, Galli M, Krauss TF. Nature Nanotechnology. 2014; 9:19-32.

14. Cheng X, Hinde E, Owen DM, Lowe SB, Reece PJ, Gaus K, Gooding JJ. Adv Mater. 2015

15. Zhong YL, Peng F, Bao F, Wang SY, Ji XY, Yang L, Su YY, Lee ST, He Y. Journal of the American Chemical Society. 2013; 135:8350-8356. [PubMed: 23581618]

16. Montalti M, Cantelli A, Battistelli G. Chemical Society Reviews. 2015; 44:4853-4921. [PubMed: 26051500]

17. Wilcoxon JP, Samara GA, Provencio PN. Physical Review B. 1999; 60:2704-2714. 
18. Baldwin RK, Pettigrew KA, Garno JC, Power PP, Liu GY, Kauzlarich SM. Journal of the American Chemical Society. 2002; 124:1150-1151. [PubMed: 11841266]

19. Heath JR. Science. 1992; 258:1131-1133. [PubMed: 17789084]

20. Mangolini L. Journal of Vacuum Science \& Technology B. 2013; 31

21. Zhong YL, Sun XT, Wang SY, Peng F, Bao F, Su YY, Li YY, Lee ST, He Y. Acs Nano. 2015; 9:5958-5967. [PubMed: 26027458]

22. Mastronardi ML, Maier-Flaig F, Faulkner D, Henderson EJ, Kubel C, Lemmer U, Ozin GA. Nano Letters. 2012; 12:337-342. [PubMed: 22195549]

23. Cullis AG, Canham LT, Calcott PDJ. Journal of Applied Physics. 1997; 82:909-965.

24. Huang K, Lu WL, Yu XG, Jin CH, Yang DR. Particle \& Particle Systems Characterization. 2016; 33:8-14.

25. Ji XY, Peng F, Zhong YL, Su YY, Jiang XX, Song CX, Yang L, Chu BB, Lee ST, He Y. Advanced Materials. 2015; 27:1029-1034. [PubMed: 25377423]

26. He Y, Kang ZH, Li QS, Tsang CHA, Fan CH, Lee ST. Angewandte Chemie-International Edition. 2009; 48:128-132.

27. Guan M, Wang WD, Henderson EJ, Dag O, Kubel C, Chakravadhanula VSK, Rinck J, Moudrakovski IL, Thomson J, McDowell J, Powell AK, Zhang HX, Ozin GA. Journal of the American Chemical Society. 2012; 134:8439-8446. [PubMed: 22509891]

28. Deubel F, Steenackers M, Garrido JA, Stutzmann M, Jordan R. Macromolecular Materials and Engineering. 2013; 298:1160-1165.

29. Pi XD, Yu T, Yang DR. Particle \& Particle Systems Characterization. 2014; 31:751-756.

30. Dasog M, Kehrle J, Rieger Bernhard, Veinot JGC. Angew Chem Int Ed Engl. 2016; 55:2322-2339. [PubMed: 26607409]

31. Mitra S, Cook S, Svrcek V, Blackley RA, Zhou W, Kovac J, Cvelbar U, Mariotti D. Journal of Physical Chemistry C. 2013; 117:23198-23207.

32. Rousseau CPF, Garcia J, Popallt M. Chem. Mater. 1995; 7:828-839.

33. Nishimura A, Harada S, Uchino T. Journal of Physical Chemistry C. 2010; 114:8568-8574.

34. Brankova T, Bekiari V, Lianos P. Chemistry of Materials. 2003; 15:1855-1859.

35. Qiao B, Wang TJ, Gao H, Jin Y. Applied Surface Science. 2015; 351:646-654.

36. Wang J, Ye DX, Liang GH, Chang J, Kong JL, Chen JY. Journal of Materials Chemistry B. 2014; 2:4338-4345.

37. Zhang XD, Chen XK, Kai SQ, Wang HY, Yang JJ, Wu FG, Chen Z. Analytical Chemistry. 2015; 87:3360-3365. [PubMed: 25671464]

38. Wu FG, Zhang XD, Kai SQ, Zhang MY, Wang HY, Myers JN, Weng YX, Liu PD, Gu N, Chen Z. Advanced Materials Interfaces. 2015; 2:1500360.

39. Liu C, Zhao Z, Zhang RL, Yang L, Wang ZY, Yang JW, Jiang HH, Han MY, Liu BH, Zhang ZP. Journal of Physical Chemistry C. 2015; 119:8266-8272.

40. Gil A, Grange P. Colloids and Surfaces a-Physicochemical and Engineering Aspects. 1996; 113:39-50.

41. Virga A, Rivolo P, Descrovi E, Chiolerio A, Digregorio G, Frascella F, Soster M, Bussolino F, Marchio S, Geobaldo F, Giorgis F. Journal of Raman Spectroscopy. 2012; 43:730-736.

42. Peng KQ, Hu JJ, Yan YJ, Wu Y, Fang H, Xu Y, Lee ST, Zhu J. Advanced Functional Materials. 2006; 16:387-394.

43. Schunemann S, Dodekatos G, Tuysuz H. Chemistry of Materials. 2015; 27:7743-7750.

44. Radziuk D, Mohwald H. Acs Nano. 2015; 9:2820-2835. [PubMed: 25704061]

45. Carlos LD, Ferreira RAS, Pereira RN, Assuncao M, Bermudez VD. Journal of Physical Chemistry B. 2004; 108:14924-14932.

46. Jakob AM, Schmedake TA. Chemistry of Materials. 2006; 18:3173-3175.

47. Wang L, Estevez MC, O'Donoghue M, Tan WH. Langmuir. 2008; 24:1635-1639. [PubMed: 18217782]

48. Green WH, Le KP, Grey J, Au TT, Sailor MJ. Science. 1997; 276:1826-1828. 
49. Shiohara A, Hanada S, Prabakar S, Fujioka K, Lim TH, Yamamoto K, Northcote PT, Tilley RD. Journal of the American Chemical Society. 2010; 132:248-253. [PubMed: 20000400]

50. Warner JH, Rubinsztein-Dunlop H, Tilley RD. Journal of Physical Chemistry B. 2005; 109:1906419067.

51. Salonen J, Kaukonen AM, Hirvonen J, Lehto VP. Journal of Pharmaceutical Sciences. 2008; 97:632-653. [PubMed: 17546667]

52. Abdalla MY. Jordan Journal of Biological Sciences. 2011; 4:119-124.

53. Zhang XD, Wu FG, Liu PD, Gu N, Chen Z. Small. 2014; 10:5170-5177. [PubMed: 25111498]

54. Herzenberg LA, DeRosa SC, Dubs JG, Roederer M, Anderson MT, Ela SW, Deresinski SC, Herzenberg LA. Proceedings of the National Academy of Sciences of the United States of America. 1997; 94:1967-1972. [PubMed: 9050888]

55. Shiomi T, Tsutsui H, Matsusaka H, Murakami K, Hayashidani S, Ikeuchi M, Wen J, Kubota T, Utsumi H, Takeshita A. Circulation. 2004; 109:544-549. [PubMed: 14744974]

56. Deng RR, Xie XJ, Vendrell M, Chang YT, Liu XG. Journal of the American Chemical Society. 2011; 133:20168-20171. [PubMed: 22107163]

57. Huang LJ, He M, Chen BB, Hu B. Journal of Materials Chemistry A. 2015; 3:11587-11595.

58. Zhang J, Yu SH. Nanoscale. 2014; 6:4096-4101. [PubMed: 24604008]

59. Wang F, Li W, Feng XL, Liu DP, Zhang Y. Chemical Science. 2016; 7:1867-1873.

60. Lehman SE, Tataurova Y, Mueller PS, Mariappan SVS, Larsen SC. Journal of Physical Chemistry C. $2014 ; 118: 29943-29951$.

61. Reid RS. Journal of Chemical Education. 1989; 66:344-345.

62. Su YY, Wei XP, Peng F, Zhong YL, Lu YM, Su S, Xu TT, Lee ST, He Y. Nano Letters. 2012; 12:1845-1850. [PubMed: 22401822]

63. Yi YH, Deng JH, Zhang YY, Li HT, Yao SZ. Chemical Communications. 2013; 49:612-614. [PubMed: 23223193] 
A
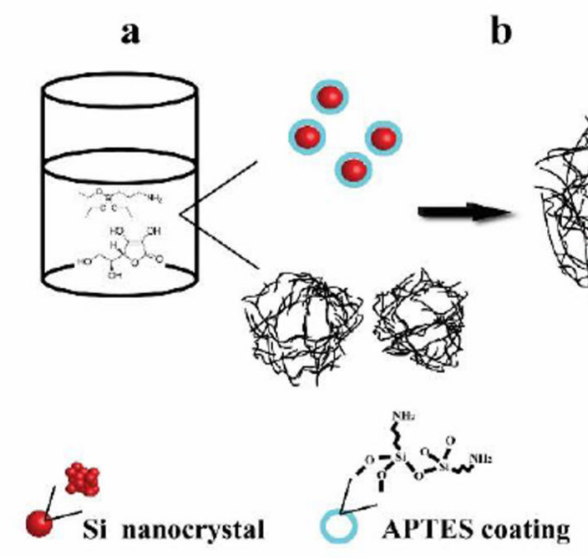

B

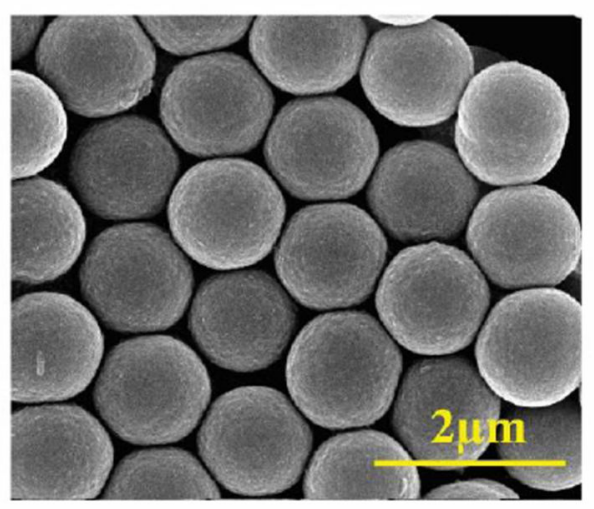

D

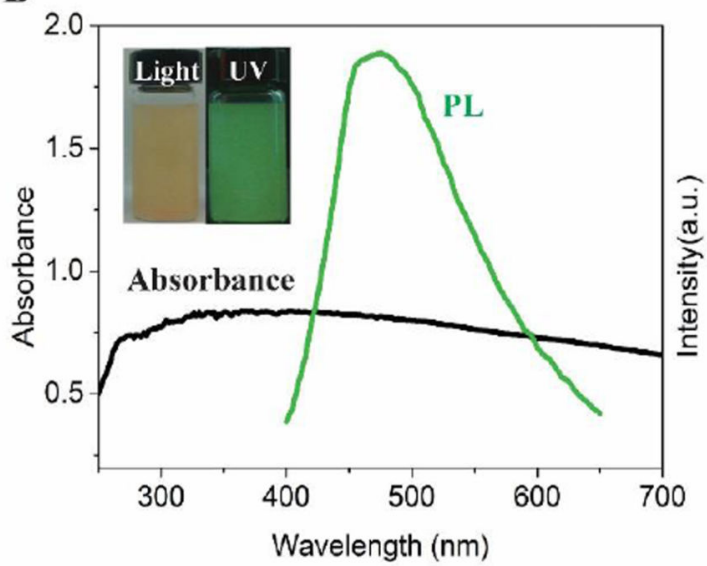

b c

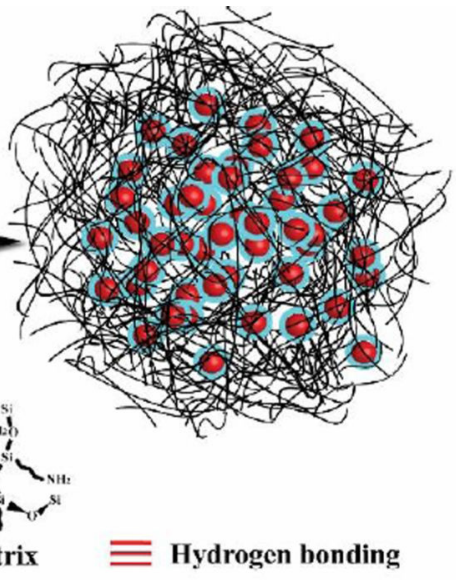

C

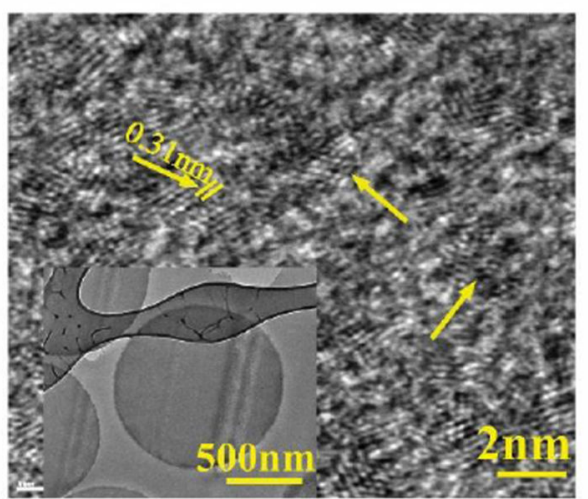

E

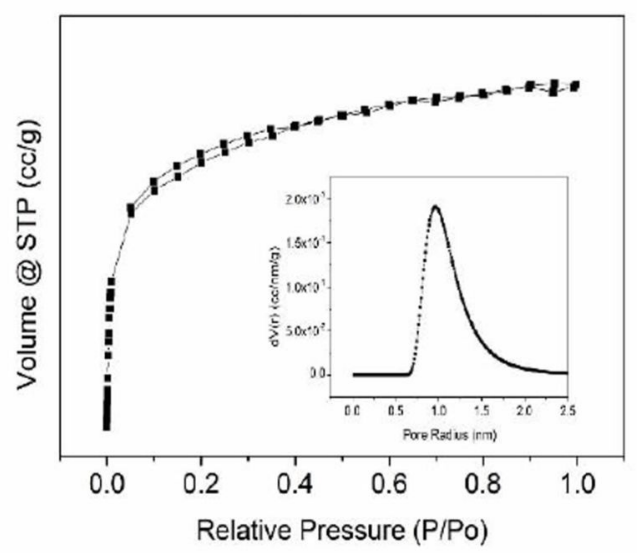

Fig. 1.

(A) Proposed scheme of one-step synthesis of SiPM a) AA reduce APTES to yield SiNC and hydrolyzed APTES form siloxance-like polymer matrices; $b$ ) formation of polymer matrices embedded with few SiNC; c) Spherical SiPM with more SiNC. (B) SEM image of typical synthetized SiPM (8 nm Pt/Pd sputtered). (C) TEM and HRTEM images of SiPM slices (70 nm thickness) cut with an ultramicrotome. (D) UV-vis absorbance and photoluminescence (PL) spectra of SiPM. Insets are picture of dispersed solution of SiPM with and without UV irradiation at $365 \mathrm{~nm}$. (E) N2 adsorption/desorption isotherms of SiPM 
(24 h reaction time), inset displays Dubinin-Astakov (DA) pore-size distribution curve obtained from the adsorption branch. 


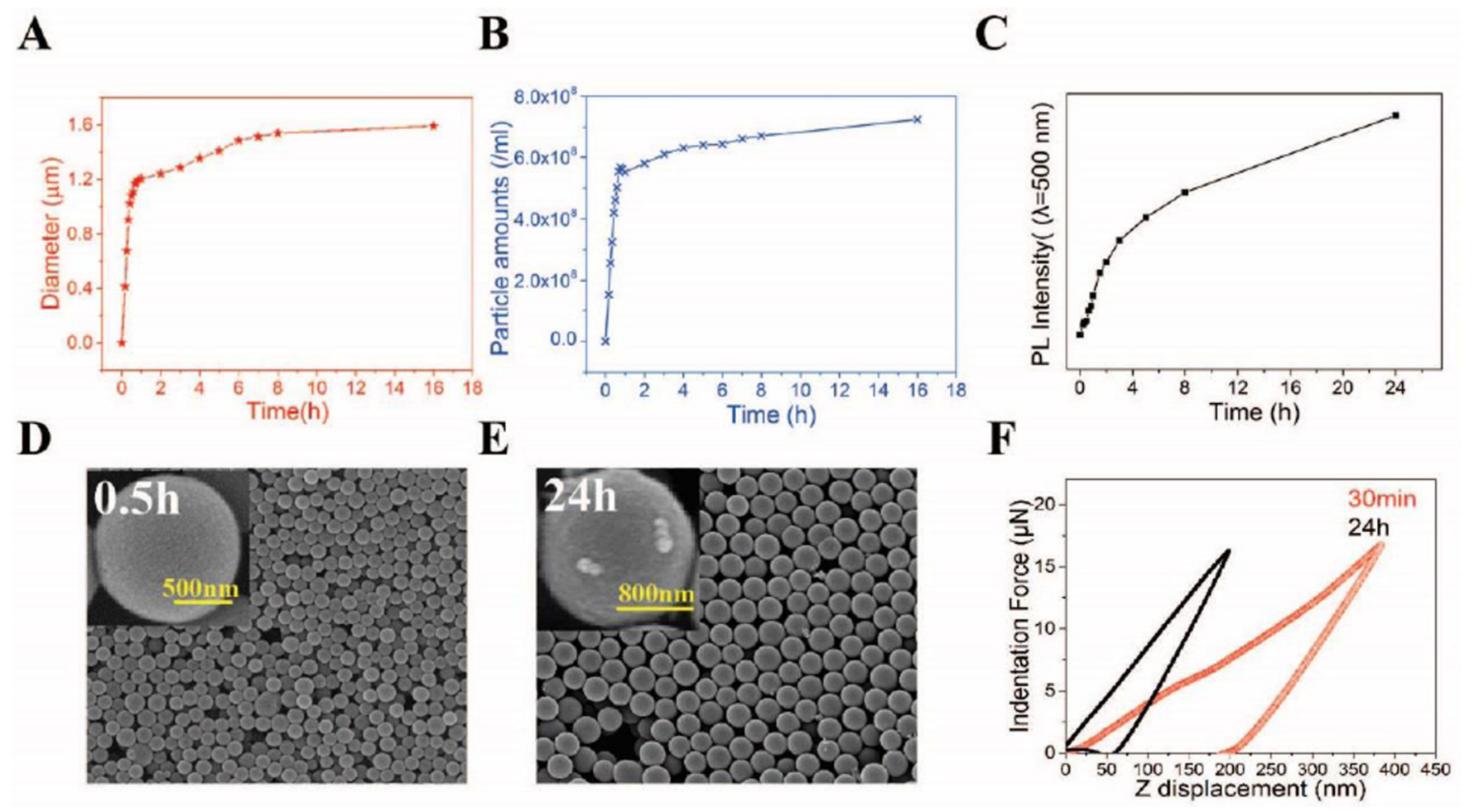

Fig. 2.

Formation process of SiPM. (A) Change of mean size, and (B) particle concentration of SiPM with reaction time. (C) The change of fluorescence intensity of SiPM at $\lambda=500 \mathrm{~nm}$ (excitation wavelength: $420 \mathrm{~nm}$ ) with reaction time. (D) SEM images of LD-SiPM (left, 30 min reaction, broader size distribution) and (E) HD-SIPM (right, $24 \mathrm{~h}$ ). (F) The force displacedment curves for the LD-SiPM and HD-SIPM under same indentation force. 

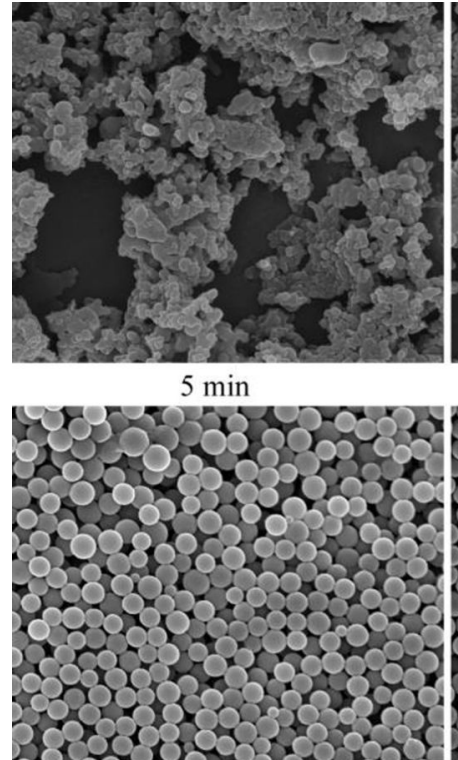

$40 \mathrm{~min}$

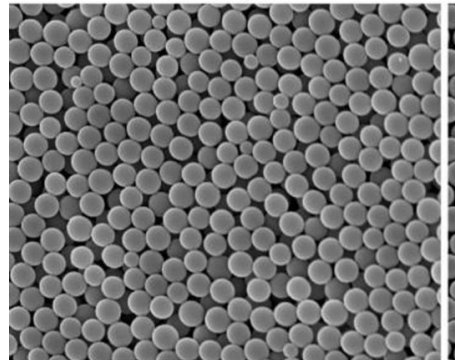

$3 \mathrm{~h}$

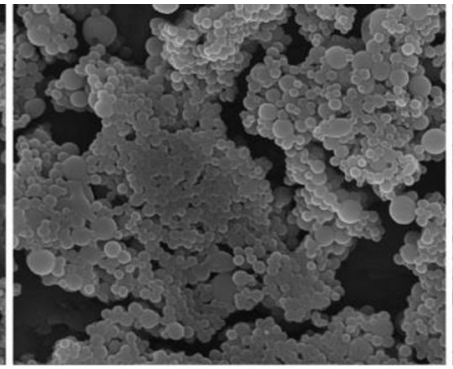

$10 \mathrm{~min}$

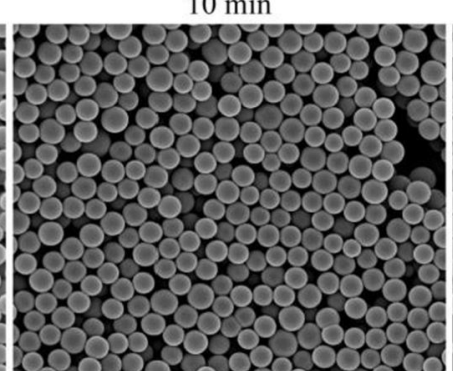

$50 \mathrm{~min}$

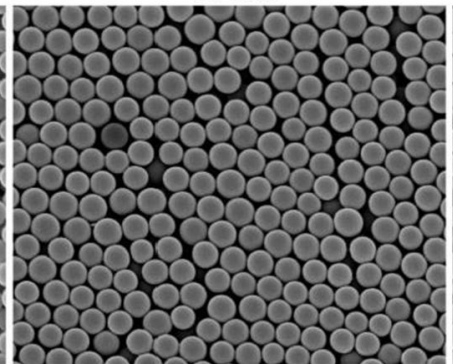

$5 \mathrm{~h}$

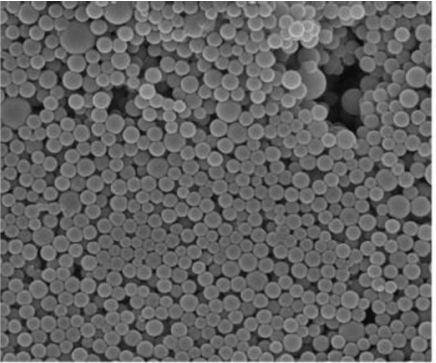

$15 \mathrm{~min}$

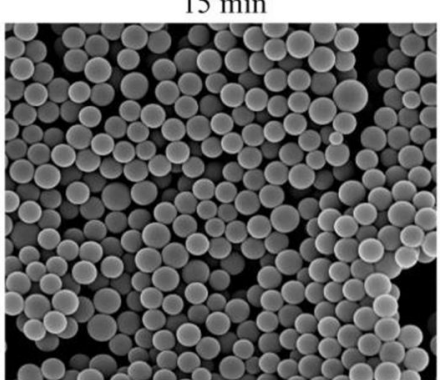

$1 \mathrm{~h}$

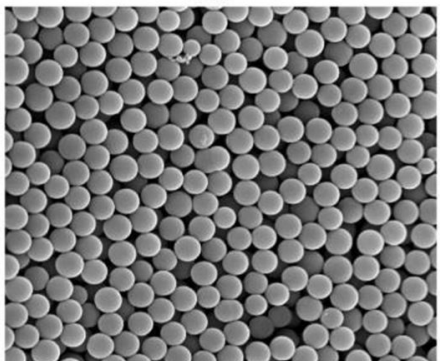

$8 \mathrm{~h}$

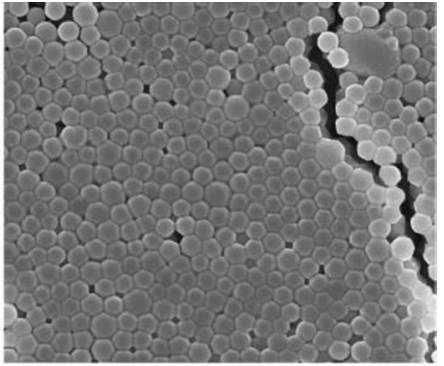

$20 \mathrm{~min}$

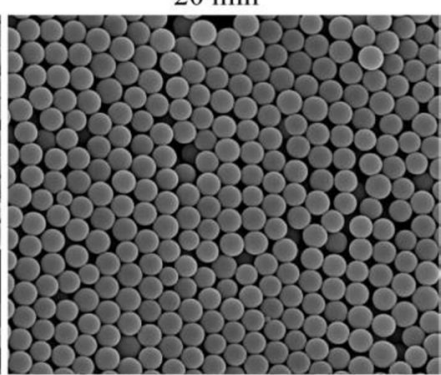

$2 \mathrm{~h}$

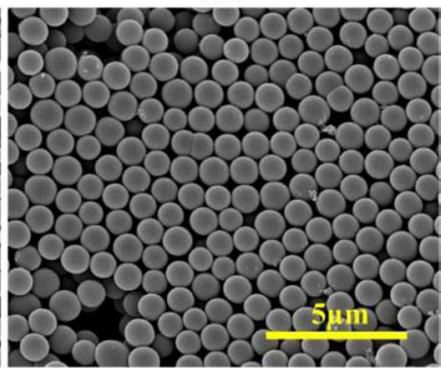

$24 \mathrm{~h}$

Fig. 3.

SEM imaging of SiPM evolution with reaction times ( $5 \mathrm{~min}-24 \mathrm{~h})$. Scale bar is $5 \mu \mathrm{m}$ for all images. 

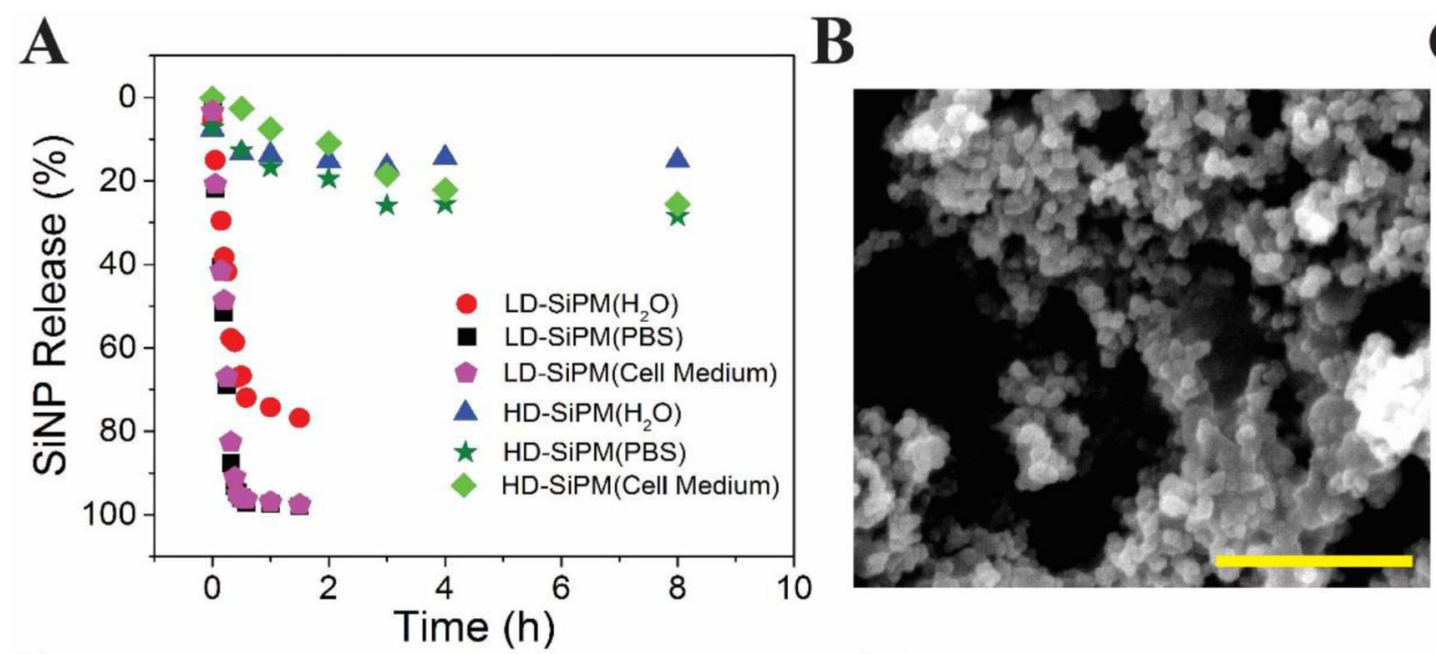

C

\section{D}
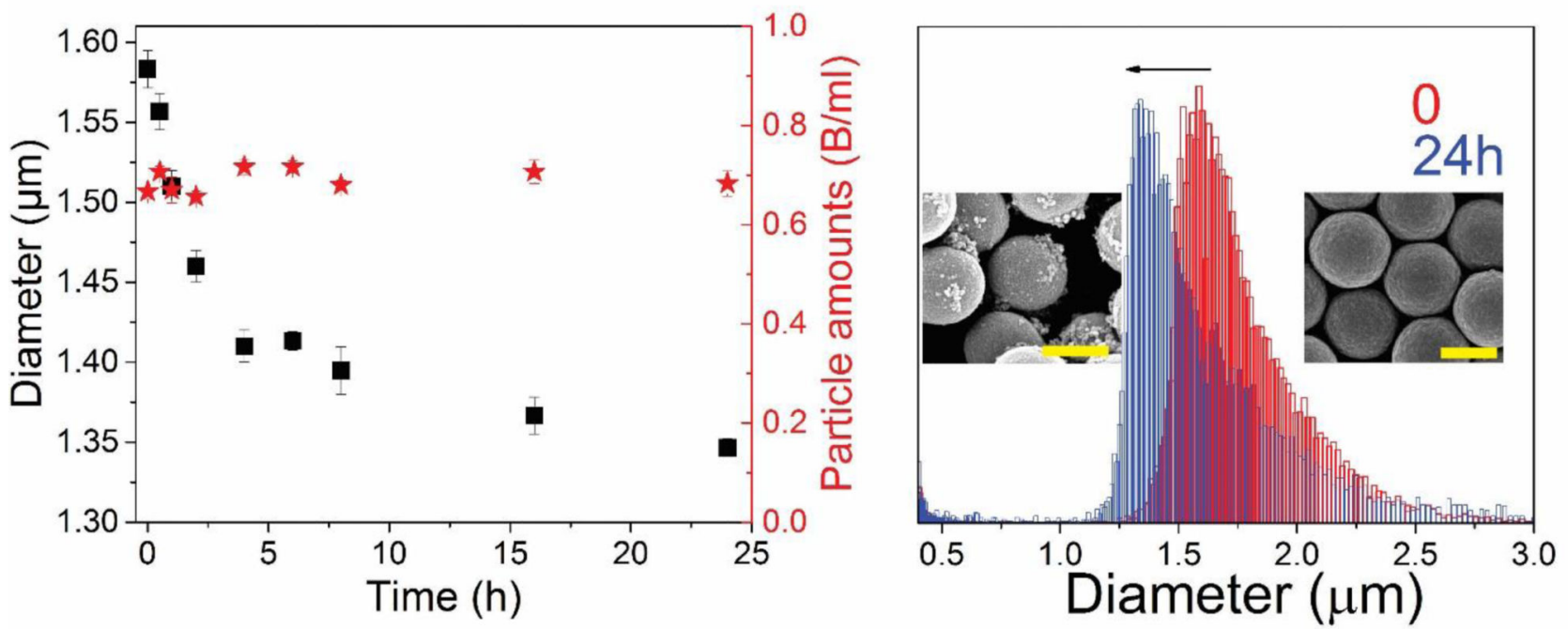

Fig. 4.

Stability of SiPM with different reaction times in aqueous media. (A) SiNP release behavior from LD-SiPM (reaction time: $30 \mathrm{~min}$ ) and HD-SiPM (reaction time: $24 \mathrm{~h}$ ) in PBS, DI water and cell culture medium. (B) SEM image of remaining SiNP/Polymer matrix from LD-SiPM in H2O. (C) Digital pictures of LD-SiPM (left, 30 min synthesis) and HD-SiPM (right, $24 \mathrm{~h}$ synthesis) immersed in PBS ( $\mathrm{pH}=7.4$ ) for $0 \mathrm{~h}$ and $24 \mathrm{~h}$. LD-SiPM sample became clear in 24 $\mathrm{h}$, indicating degraded substantial degradation, while HD-SiPM sample showed little change. (D) Change of diameter and amount of HD-SiPM along with time in PBS. (E) Multisizer results of size distribution of HD-SiPM at beginning and after $24 \mathrm{~h}$ immersion in PBS. Insets are SEM images of related HD-SiPM. Scale bar is $1 \mu \mathrm{m}$ for all images. 
A

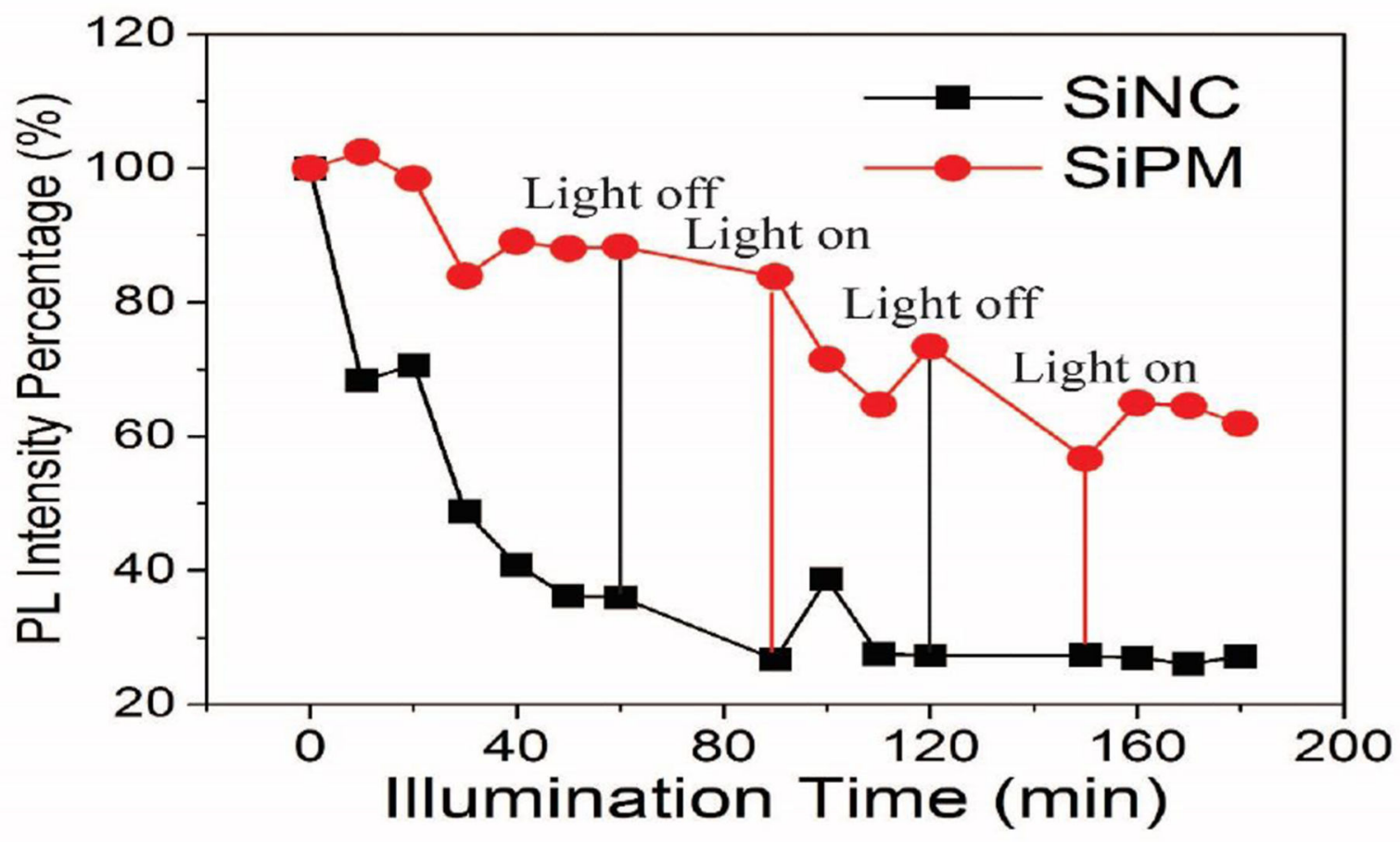

B

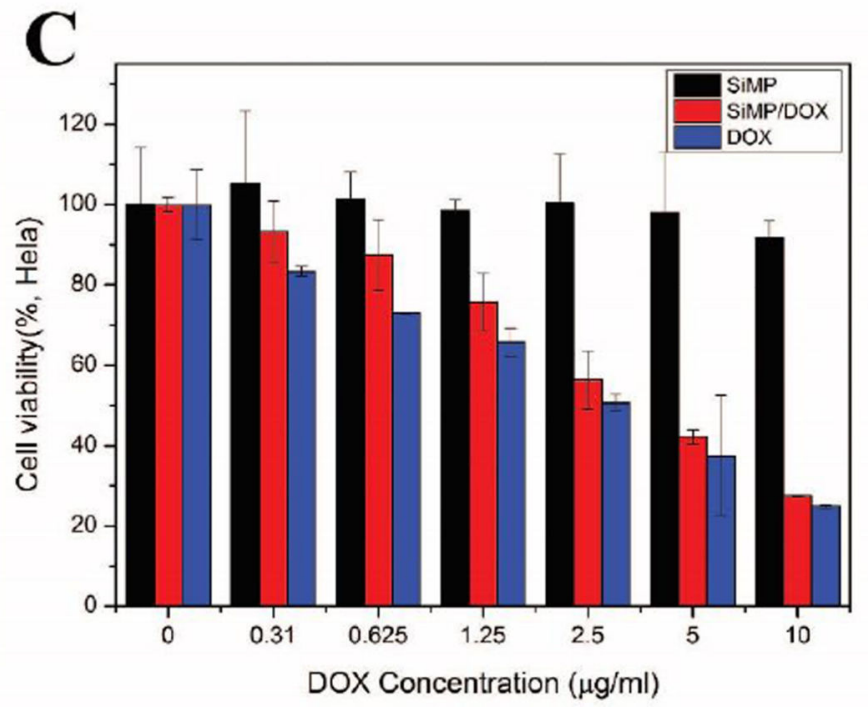

Fig. 5.

(A) Remained PL emissions of Si nanoparticles and SiPM solutions along with increasing illumination time. (Rayonet-600 photochemical reactor, 120W) (B) 3D confocal image of Hela cells after incubated with SiPM for $4 \mathrm{~h}$. (C) Cell viability of Hela cells after $24 \mathrm{~h}$ incubation with free DOX, SiMP and SiMP/DOX of various concentrations DOX. Results are the average from three independent experiments. 
A

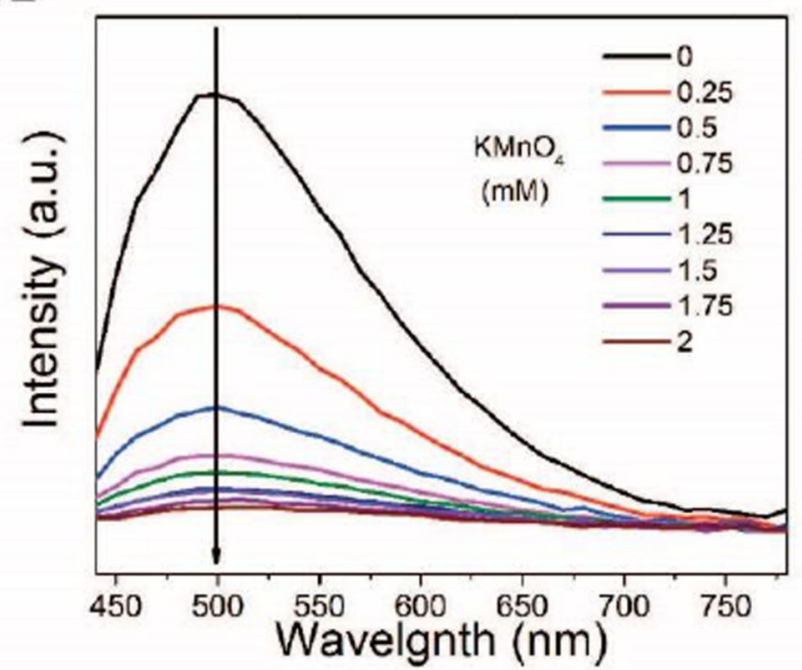

C

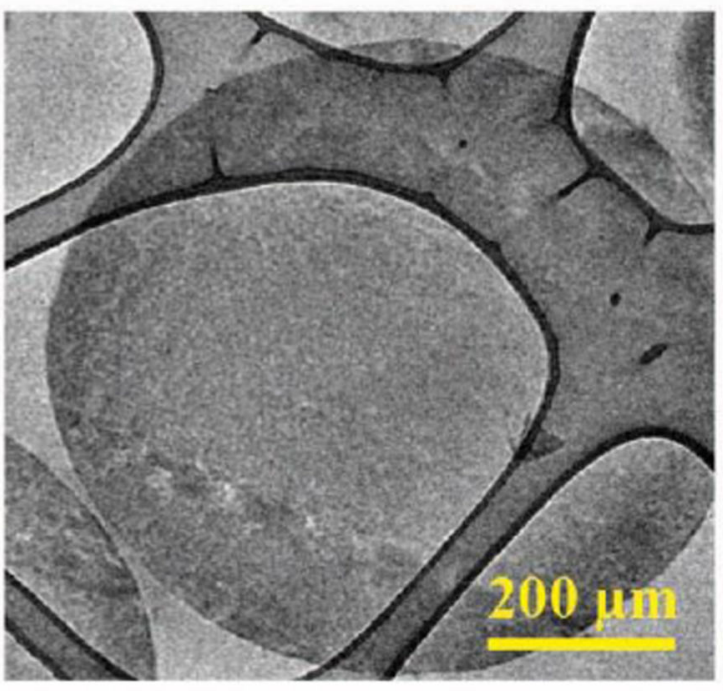

B

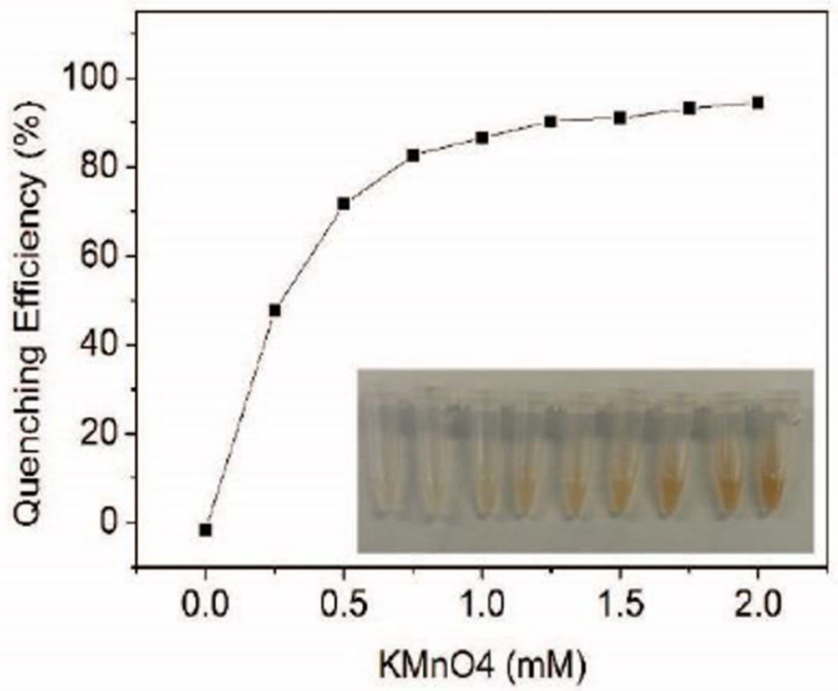

D

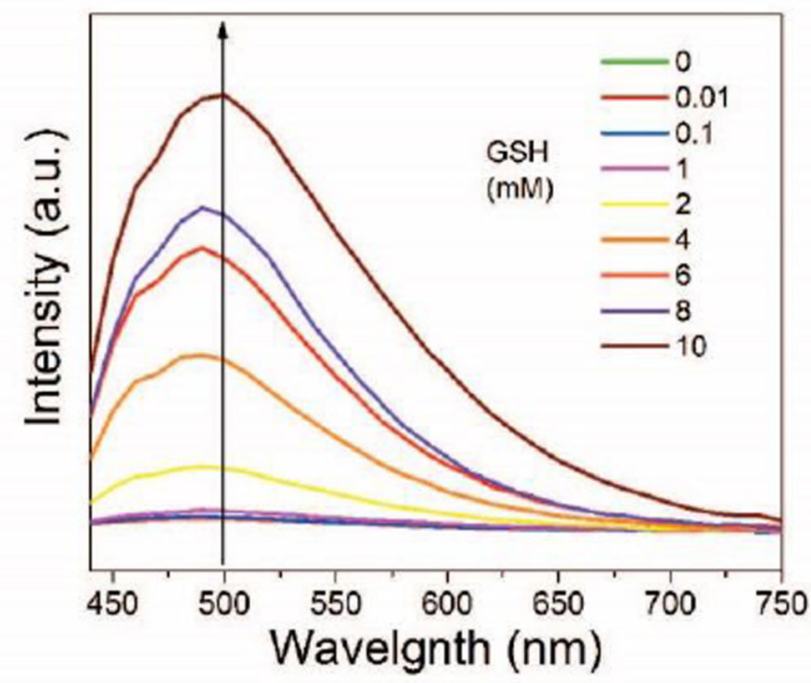

Fig. 6.

Bio-sensing by using $\mathrm{SiPM} / \mathrm{MnO}_{2}$ composite. (A) Photolumunescence spectra (excitation wavelength: $420 \mathrm{~nm}$ ) of $\mathrm{SiPM} / \mathrm{MnO}_{2}$ resulted from various concentration of $\mathrm{KMnO}_{4}$ in reaction. (B) The quenching efficiency of $\mathrm{MnO}_{2}$ on SiPM along with increasing concentration of $\mathrm{KMnO}_{4}$, inset is captured images of corresponding $\mathrm{SiPM} / \mathrm{MnO}_{2}$ composites. (C) TEM image of SiPM/MnO 2 composite slices (thickness: $70 \mathrm{~nm}$ ) (D) The fluorescence spectra (excitation wavelength: $420 \mathrm{~nm})$ of $\mathrm{SiPM} / \mathrm{MnO}_{2}(\mathrm{SiPM}$ reacted with 2 $\mathrm{mM} \mathrm{KMnO} 4$ ) with increasing GSH concentration. 


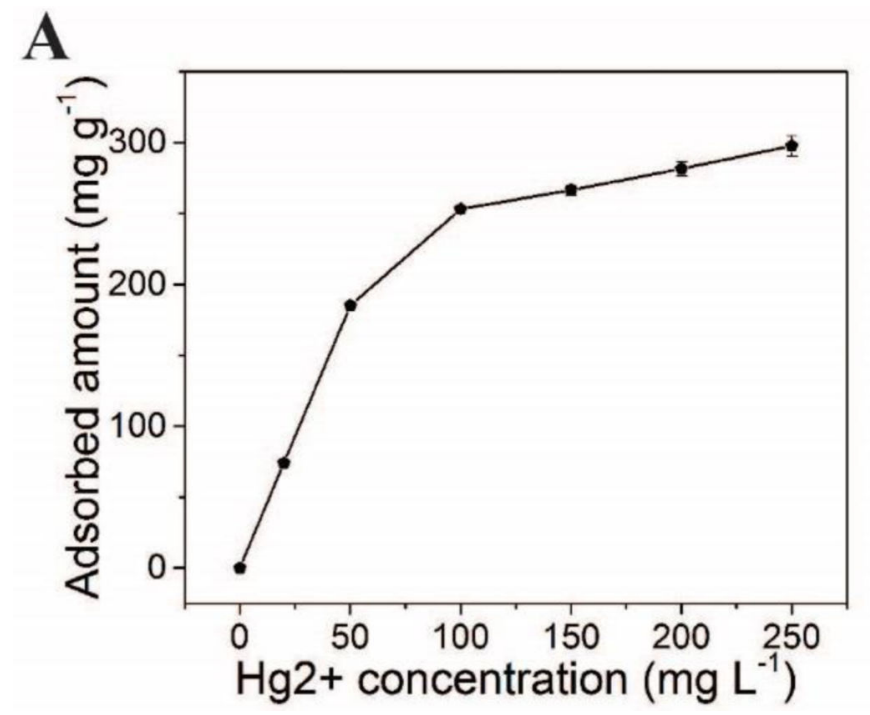

B

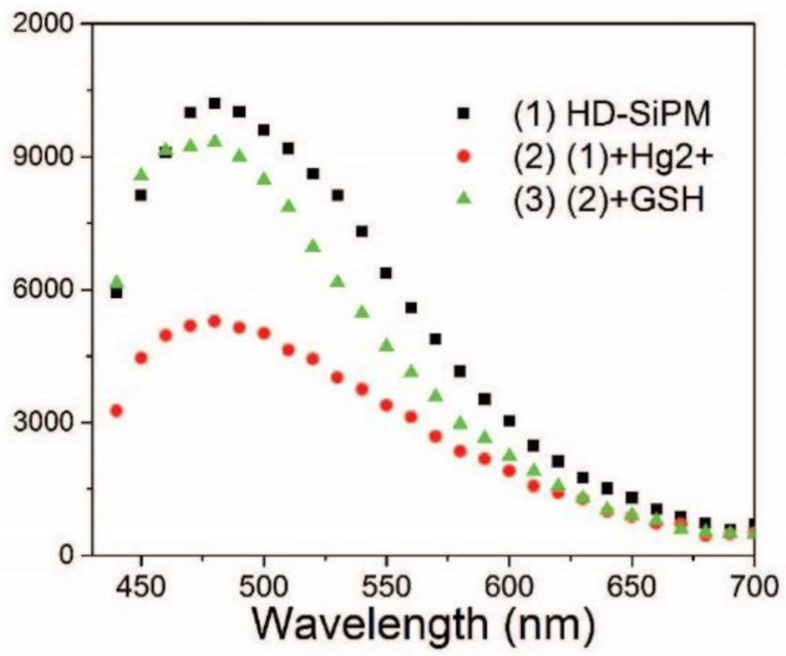

Fig. 7.

(A) Adsorption curve of $\mathrm{Hg}^{2+}$ at various concentrations by using HD-SiPM. (B) the fluorescent spectra (excitation wavelength: $420 \mathrm{~nm}$ ) of SiPM before (black) and after addition of $\mathrm{Hg}^{2+}(1 \mathrm{mM}$, red) and after addition of GSH (green). 
A

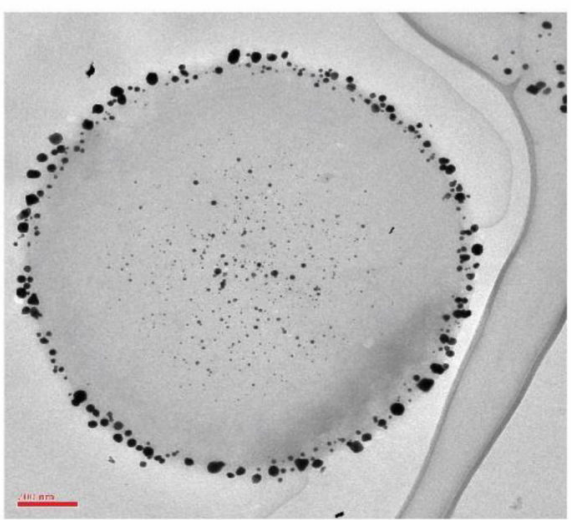

$\mathrm{C}$

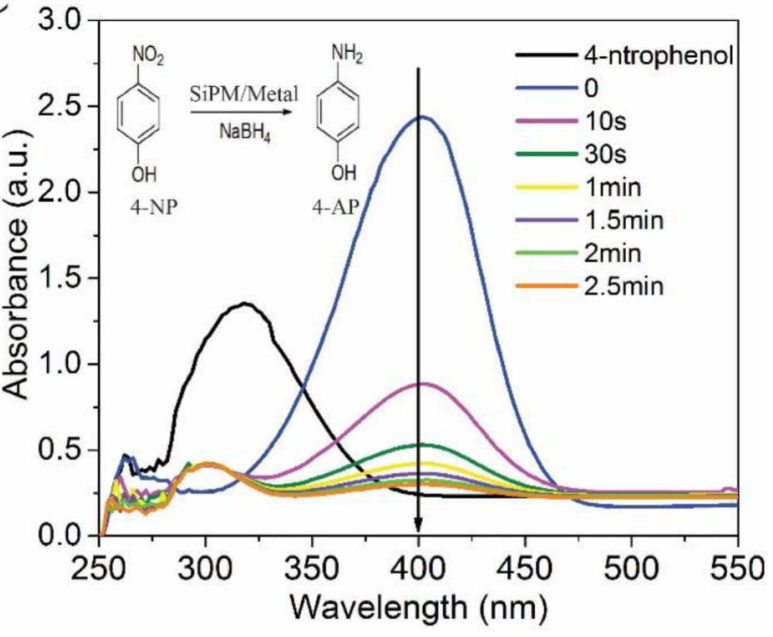

B

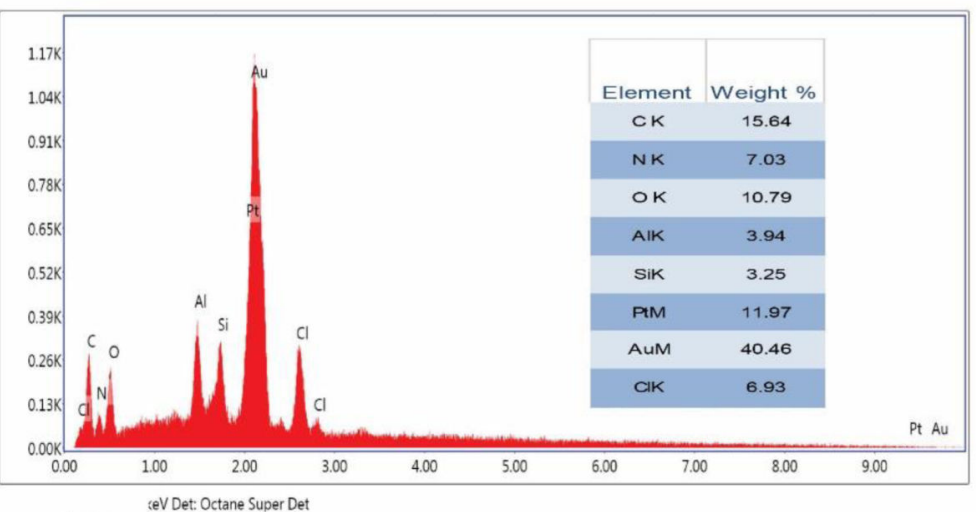

D

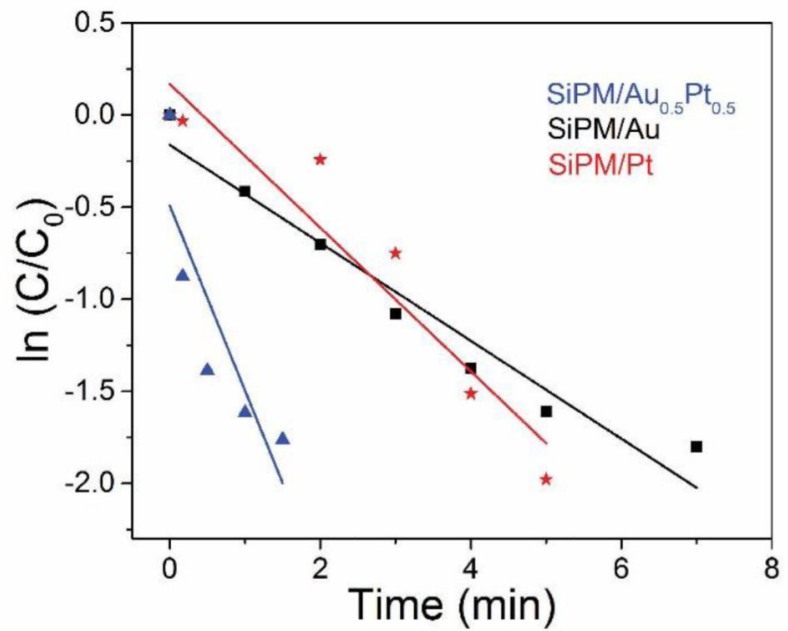

Fig. 8.

(A) TEM image of SiPM/AuNP slices (70 $\mathrm{nm}$ thin), indicating SiPM reduced gold ions to form AuNP (dark-phased black particles) throughout SiPM matrix. (B) EDS spectrum of $\mathrm{SiPM} / \mathrm{Au}_{0.5} / \mathrm{Pt}_{0.5}$, inset is elements composition. (C) UV-vis spectra during catalytic conversion of 4-NP by SiPM/Au $0.5 / \mathrm{Pt}_{0.5}$ over time, inset is principle of reduction of 4-NP by $\mathrm{NaBH}_{4}$ to form 4-AP (D) $\mathrm{Ln}\left(\mathrm{C} / \mathrm{C}_{0}\right)$ versus $\mathrm{t}$ for the reduction of 4-NP catalyzed by $\mathrm{SiPM} / \mathrm{Au}, \mathrm{SiPM} / \mathrm{Pt}$ and $\mathrm{SiPM} / \mathrm{Au}_{0.5} / \mathrm{Pt}_{0.5}$. 

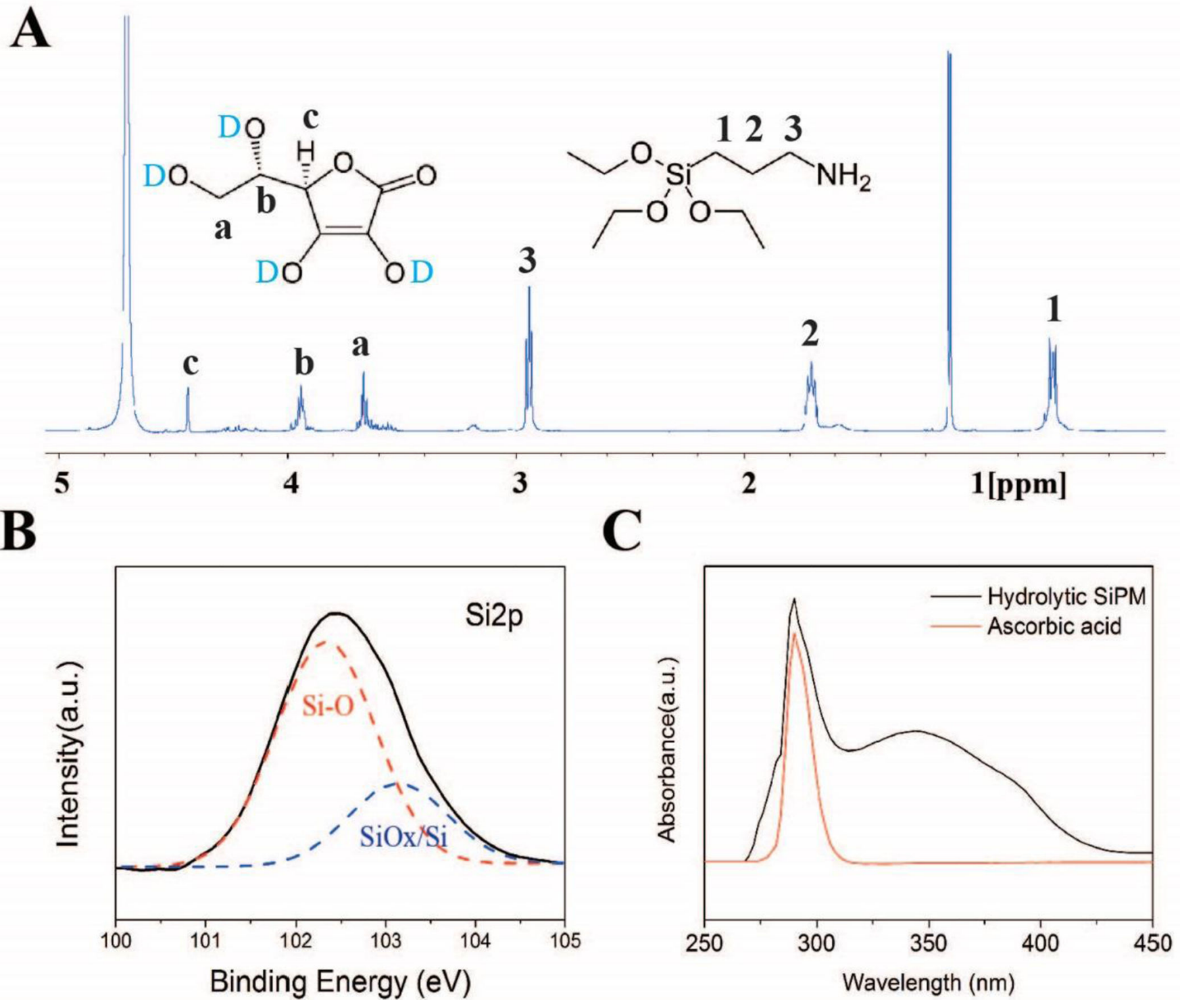

Fig. 9.

(A) Solution-phase 1D 1H NMR spectrum of hydrolytic SiPM in $\mathrm{D}_{2} \mathrm{O}$. (B) Structure analysis of high resolution XPS spectra: $\mathrm{Si} 2 \mathrm{p}$. (C) UV-vis absorption spectrum of hydrolytic SiPM and L-ascorbic acid. 